\title{
Article \\ Sign Stability of Dual Switching Linear Continuous-Time Positive Systems
}

\author{
Liang Liu ${ }^{1}$, Fei Long ${ }^{2,3, *}$, Lipo Mo ${ }^{4}$ and Qianqian $\mathrm{Mu}^{5}$ \\ 1 College of Electrical Engineering, Guizhou University, Guiyang 550025, China; gs.liangliu19@gzu.edu.cn \\ 2 College of Artificial Intelligence and Electrical Engineering, Guizhou Institute of Technology, \\ Guiyang 550003, China \\ 3 Special Key Laboratory of Artificial Intelligence and Intelligent Control of Guizhou Province, \\ Guiyang 550003, China \\ 4 School of Mathematics and Statistics, Beijing Technology and Business University, Beijing 100048, China; \\ molipo@th.btut.edu.cn \\ 5 School of Mathematics and Big Data, Guizhou Education University, Guiyang 550018, China; \\ gs.qqmu19@gzu.edu.cn \\ * Correspondence: feilong@git.edu.cn
}

Citation: Liu, L.; Long, F.; Mo, L.; $\mathrm{Mu}, \mathrm{Q}$. Sign Stability of Dual Switching Linear Continuous-Time Positive Systems. Symmetry 2021, 13, 2194. https://doi.org/10.3390/ sym13112194

Academic Editor: Jan Awrejcewicz

Received: 23 September 2021

Accepted: 10 November 2021

Published: 17 November 2021

Publisher's Note: MDPI stays neutral with regard to jurisdictional claims in published maps and institutional affiliations.

Copyright: ( $\odot 2021$ by the authors Licensee MDPI, Basel, Switzerland. This article is an open access article distributed under the terms and conditions of the Creative Commons Attribution (CC BY) license (https:// creativecommons.org/licenses/by/ $4.0 /)$.

\begin{abstract}
This paper investigates 1-moment exponential stability and exponential mean-square stability (EMS stability) under average dwell time (ADT) and the preset deterministic switching mechanism of dual switching linear continuous-time positive systems when a numerical realization does not exist. The signs of subsystem matrices, but not their structures of magnitude, are key information that causes a qualitative concept of stability called sign stability. Both 1-moment exponential stability and EMS stability, which are the traditional stability concepts, are generalized intrinsically. Hence, both 1-moment exponential sign stability and EMS sign stability are introduced and are proven based on sign equivalency. It is shown that they are symmetrically and qualitatively stable. Notably, the notion of stability can be checked quantitatively using some examples.
\end{abstract}

Keywords: 1-moment exponential stability; exponential mean-square stability; sign stability; dual switching linear continuous-time positive systems; symmetrical qualitative stability

\section{Introduction}

Hybrid systems, which are classified based on probabilistic behavior and different driving switching mechanisms, are employed in a wide range of implementations. They are composed of the coexistence of both discrete-time and continuous-time dynamics, which have been investigated in the literature. They are referred to as deterministic switching systems [1] and stochastic switching systems [2], respectively. For example, an automatic shifting car is a switching strategy that can be controlled and designed by a deterministic switching system. The ADT and the preset deterministic switching strategy are common methods for studying deterministic switching [3-5]. The characterization of a stochastic switching system is such that its subsystems obey a random process. The random process provides a method for time-varying network modeling via Markovian random graph processes [6]. The Markov jump system, a widely implemented stochastic switching system, is based on a switching strategy defined by a Markov process. $\delta$-moment, exponentially mean-square (EMS), and exponentially almost-sure (EAS) are different stability improvements generated by the deterministic and the stochastic switching systems [7-9]. Dual switching systems [10], defined as a combination of a switching strategy based on deterministic approaches and a switching signal based on stochastic approaches, cause "jumps" at arbitrary occasions, while the switching defined stochastically is not affected by deterministic switching. For example, a connection is established between a wind turbine and some energy accumulator equipment [11]. A switching signal defined deterministically 
could reasonably find shifts between working modes of the turbine, while an appropriate controller determines its timetable. Nevertheless, external factors affecting the wind generation system are related to the shifts between the modes of charging equipment and could be defined by a stochastic model. Furthermore, the invariance property of positive linear systems implies the nonnegative orthant [12]. The switched positive system is a special kind of switched system, which is composed of finite positive subsystems and switching strategies. In [13], it shows that a switched linear system is $m$ th mean stable under a positive system. Furthermore, the positive system has broad applications in many fields, e.g., communication networks [6,14], formation flying [15], etc.

Although the dual switching system solves many complex control engineering problems, obtaining numerical matrices satisfying the linear matrix inequality (LMI) constraints of the stability of a dual switching system is difficult, which results in the growth of robust control theory. A quantitative matrix can be transformed into a qualitative matrix by utilizing robust control approaches. Hence, a nominal model with finite changes in its vicinity is considered. Although the magnitudes of interconnections among the states cannot be predicted reliably, their corresponding signs could be found solidly. Therefore, a concept called sign stability was introduced to analyze dual switching linear systems represented by matrices whose negative eigenvalues are observed in their sign structure.

A certain matrix whose elements follow a sign pattern consists of the sign $\{+,-, 0\}$. The sign stability property of a matrix can be expressed if an arbitrary matrix follows the sign pattern similar to that of the original matrix and has a Hurwitz stability, regardless of the values of its elements $[16,17]$. The sign stability notion was first suggested in bionomics and utilized to assess the interactions between various large scaled ecosystem categories that cannot be defined by solid models and lofty sturdiness and various other types of uncertainties. The state-space matrix's entity denoted by $(i, j)$ is represented by signs $\{+,-, 0\}$. While a positive sign is related to the class $j$, which has a positive effect on the class $i$. A negative effect is designated by a negative sign. No effect is denoted by zero. A visual description of the aforementioned situation could be provided by a directed graph, which is a graph reflecting the positive or negative relationship of the elements of the sign pattern matrix. For example, assuming that the corresponding sign pattern matrices, called directed graphs, are denoted by the following:

$$
\operatorname{sgn}(A)=\left[\begin{array}{ccc}
0 & + & + \\
- & 0 & 0 \\
- & 0 & -
\end{array}\right]
$$

Figure 1 depicts $\boldsymbol{A}^{\prime}$ s directed graph, so Figure 1 is called the directed graph of $\operatorname{sgn}(\boldsymbol{A})$.

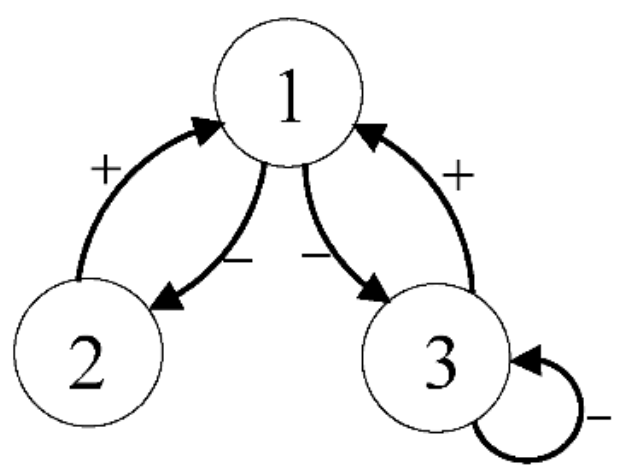

Figure 1. $A^{\prime}$ s directed graph.

Many researchers began to analyze sign stability and achieved great research results. Sufficiency and necessity requirements concerning the sign stability of matrices are derived by Jeffries, and the color test method to examine the sign stability for a given matrix in ecological terms is proposed [18]. Nevertheless, the color test conditions are quite 
complicated for engineering problems. New necessary and sufficient conditions for sign stability were derived by some researchers $[19,20]$. Control engineering, particularly for designing robust controllers, is an area in which the sign stability approach could be employed, which is suggested by theoretical researchers. More robustness could be reached by the aforementioned approach and conservativeness is lowered when compared to the traditional Lyapunov-based stability approaches. Wang and Dong implemented the sign stability to some uncertain systems, such as flight control and switching systems, to extract sufficient and necessary requirements that lead to the sign stability concerning switched systems under a defined switching strategy [21,22]. An ecological sign-stability approach to construct a robust controller for linear systems was adopted by Rama who employed the sign-stability idea from ecology to develop a novel control design strategy for uncertain linear systems with robustness to a category of uncertainties in the plant parameters [23-25]. Sign stability of linear systems is characterized by a positive Markov jump verified by sign stability to stochastic switching systems, which was applied by Cavalcanti [26,27].

The sign stability of dual switching linear positive systems (DSLPSs) is verified by the current research, its main contributions are: (i) the extraction of sufficient conditions so the stability of a DSLPS is guaranteed; (ii) 1-moment exponential sign stability and EMS sign stability of DSLPSs are verified. Firstly, the paper analyzes 1-moment exponential and EMS sign stability of dual switching linear continuous-time positive systems (DSLCTPSs), which depend upon the ADT and the predetermined deterministic switching strategy. Sufficiency conditions represented by LMI are then given. The sign stability analysis of DSLPS cannot be utilized by the previous strategy herein since each subsystem matrix is stable, which is not enough and is not necessary for the stability of DSLPSs. The equivalence between the stability of DSLPSs and particular matrices through LMI are also designated by our results that are similar to our previous research. Qualitative results can be of merit and an intuitive complement to conventional numerically based methods could be employed for robust stability analysis.

\section{Preliminaries}

The work is organized as follows. In Section 2.1, we introduce some related mathematical concepts. In Section 2.2, we first define the mathematical model of DSLCTPSs, 1-moment exponential stability, and EMS stability. We then use ADT and the preset deterministic switching strategy to obtain the sufficient conditions of the two kinds of stability from the sufficient condition that starts with the analysis of the equivalent condition of the corresponding sign stability. In Section 2.3, we finally define the sign stability of DSLCTPSs.

\subsection{Notation}

$\boldsymbol{R}_{n \times n}$ represents the set of real matrix of dimension $n$, and the $n$-dimensional identity matrix is denoted by $\boldsymbol{I}_{n}$. For matrix $\boldsymbol{A}_{n \times n}=\left(a_{i j}\right)_{n \times n^{\prime}} \boldsymbol{A}_{i, j}$ denotes the element $a_{i j}$ of $\boldsymbol{A}$, the diagonal elements $\left\{a_{11}, a_{22}, \ldots, a_{n n}\right\}$ of $A$ are denoted by $\operatorname{diag} A_{n \times n}$. Let $\boldsymbol{A}_{i}, i=1,2, \ldots, n$ be matrices and let blockdiag $\left(A_{1}, \ldots, A_{n}\right)$ be a matrix whose diagonal elements are called block diagonal. $A_{1}$ is called the upper left block, the other diagonal block is called $A_{2}$, and $A_{n}$ is called a bottom right block. Let $\boldsymbol{e}_{i}$ define a column vector whose elements equal 0 excluding the $i$ th one that is either 1 or " + ". The other vector defined by a particular notation is 1 , where all elements are 1 . $\operatorname{vec}(\boldsymbol{X})$ denotes the expansion of the column vector of $X . \operatorname{sgn}(a)$ designates the sign of $a . a$ and $b$ having the same sign is denoted by equality $\operatorname{sgn}(a)=\operatorname{sgn}(b) . G_{A}$ denotes the directed graph of $\operatorname{sgn} A_{n \times m}$. For a random variable $x(t)$, its expected value is denoted by $\mathrm{E}(\boldsymbol{x}(t))$. $\boldsymbol{A}$ and $\boldsymbol{B}$ are two square matrices whose Kronecker's product and Kronecker's summation are expressed respectively by the following:

$$
\boldsymbol{A} \otimes \boldsymbol{B}=\left[\begin{array}{ccc}
a_{11} \boldsymbol{B} & \ldots & a_{1 n} \boldsymbol{B} \\
\vdots & \ddots & \vdots \\
a_{n 1} \boldsymbol{B} & \ldots & a_{n n} \boldsymbol{B}
\end{array}\right]
$$


and

$$
\boldsymbol{A} \oplus \boldsymbol{B}=\boldsymbol{A} \otimes \boldsymbol{I}_{m}+\boldsymbol{I}_{n} \otimes \boldsymbol{B} .
$$

2.2. Stability of Dual Switching Linear Continuous-Time Positive Systems (DSLCTPSs)

Assume that a DSLCTPS is defined by

$$
\left\{\begin{array}{l}
\dot{x}(t)=A_{\sigma(t, \gamma(t))}^{[\gamma(t)]} x(t), t>0 \\
x(0)=x_{0}
\end{array}\right.
$$

where the continuous state denoted by $x(t)$ is described by a real $n$-dimensional vector, a real Metzler matrix whose dimension is $n\left(A_{i}^{[j]}\right.$ having nonnegative values on each off diagonal) is denoted by $A_{i}^{[j]}$, such that $x(0)$ is positive, $x(t)$ evolves in the positive orthant. $\gamma(\cdot):[0,+\infty) \rightarrow \overline{\mathrm{M}}, \overline{\mathrm{M}}=\{1,2, \ldots, \mathrm{M}\}, \mathrm{M}<+\infty$ denotes the deterministic switching signal whose functional form is constant and piecewise right continuous where the index of the active Markov subsystem is indicated. $\gamma(t)=\gamma_{k} \in \overline{\mathrm{M}}$ for $t \in\left[t_{k}, t_{k+1}\right)$, where switching time at instant $k$ is denoted by $t_{k}$, where $k \in \overline{\mathrm{N}}$ and $t_{0}=0$. The switching signal defined by $\sigma(\cdot, \cdot):[0,+\infty) \times \overline{\mathrm{M}} \rightarrow \overline{\mathrm{N}}, \overline{\mathrm{N}}=\{1,2, \ldots, \mathrm{N}\}, \mathrm{N}<+\infty$ denotes a function that is piecewise and constant and it is governed by an N-mode Markov process. The subscript of the active subsystem expressed by $\sigma\left(t, \gamma_{k}\right)=\sigma_{v}^{\gamma_{k}} \in \overline{\mathrm{N}}$ for $t \in\left[\tau_{v}, \tau_{v+1}\right) \subset\left[t_{k}, t_{k+1}\right)$ is defined where $\tau_{v}$ denotes the switching time at the instance $v, v \in\{0,1,2, \ldots, l\}, l<\infty$ and $\tau_{0}=t_{k}, \tau_{l+1} \leq t_{k+1}$. For every $j \in \overline{\mathrm{M}}, \sigma(t, j)$, called the Markov process transition probability, is defined by

$$
p_{r s}^{[j]}(\Delta)=\operatorname{Pr}\{\sigma(u+\Delta, j)=s \mid \sigma(u, j)=r\}=\left\{\begin{array}{c}
q_{r s}^{[j]} \Delta+(\Delta), r \neq s \\
1+q_{r r}^{[j]} \Delta+(\Delta), r=s
\end{array}\right.
$$

when $r, s \in \overline{\mathrm{N}}, \Delta>0$ and $\lim _{\Delta \rightarrow 0}(\Delta) / \Delta=0, q_{r s}^{[j]}$ defines the transition rate from mode $r$ and mode $s$ at the times $u$ and $u+\Delta$, respectively

$$
\left\{\begin{array}{l}
q_{r s}^{[j]} \geq 0, s \neq r \\
\sum_{s=1, s \neq r}^{\mathrm{N}} q_{r s}^{[j]}=-q_{r r}^{[j]}
\end{array}\right.
$$

where $Q^{[j]}=\left[q_{r s}^{[j]}\right]_{\mathrm{N} \times \mathrm{N}}$ denotes the transition rate matrix of $\sigma(t, j) . \sigma(t, j)$, called the Markov process, is considered irreducible if $\forall j \in \bar{M}$. Thus, it is called ergodic and its unique invariant distribution $\boldsymbol{\Pi}^{[j]}=\left[\pi_{1}^{[j]}, \pi_{2}^{[j]}, \ldots, \pi_{N}^{[j]}\right]$ satisfies

$$
\left\{\begin{array}{c}
\Pi^{[j]} \boldsymbol{Q}^{[j]}=0 \\
\sum_{i=1}^{\mathrm{N}} \pi_{i}^{[j]}=1
\end{array}\right.
$$

The increased time stays in the subsystem of $\dot{x}(t)=A_{i}^{[j]} x(t)$ in $[0, t]$ and is denoted by $T_{i}^{[j]}(0, t)$ and the subsystem's incident number of $\dot{x}(t)=A_{i}^{[j]} x(t)$ in $[0, t]$ is denoted by $N_{i}^{[j]}(0, t)$.

Lemma 1. (Ref. [8]) For any $j \in \overline{\mathrm{M}}, \sigma(t, j)$, an $N$-mode Markov process for $[0, t)$ is assumed. The Markov process $\sigma(t, j)$ whose transition rate matrix and stationary distribution are denoted by $\boldsymbol{Q}^{[j]}=\left[q_{r s}^{[j]}\right]_{N \times N}$ and $\Pi^{[j]}=\left[\pi_{1}^{[j]}, \pi_{2}^{[j]}, \ldots, \pi_{N}^{[j]}\right]$, respectively. Then, for $\forall i \in \overline{\mathrm{N}}$ and $\forall j \in \overline{\mathrm{M}}$, the probability equation is defined by

$$
P\left\{\lim _{t \rightarrow \infty} \frac{T_{i}^{[j]}(0, t)}{t}=\pi_{i}^{[j]}\right\}=1
$$


and

$$
P\left\{\lim _{t \rightarrow \infty} \frac{N_{i}^{[j]}(0, t)}{t}=\pi_{i}^{[j]}\left|q_{i i}^{[j]}\right|\right\}=1
$$

Remark 1. For $\forall i \in \overline{\mathrm{N}}, \forall j \in \overline{\mathrm{M}}$, there is a positive constant $\varepsilon$ that meets

$$
T_{i}^{[j]}(0, t) \leq\left(\pi_{i}^{[j]}+\varepsilon\right) t
$$

and

$$
N_{i}^{[j]}(0, t) \leq\left(\pi_{i}^{[j]}\left|q_{i i}^{[j]}\right|+\varepsilon\right) t
$$

Proof. From (6), $\forall \varepsilon>0, \exists T$, while $t>T,\left|\frac{T_{i}^{[j]}(0, t)}{t}-\pi_{i}^{[j]}\right| \leq \varepsilon$, as shown in Figure 2, then $\pi_{i}^{[j]}-\varepsilon \leq \frac{T_{i}^{[j]}(0, t)}{t} \leq \pi_{i}^{[j]}+\varepsilon$. For different $T$, there are corresponding $\varepsilon$, so while $t>T$, $T_{i}^{[j]}(0, t) \leq\left(\pi_{i}^{[j]}+\varepsilon\right) t$. The same is true for $(8)$.

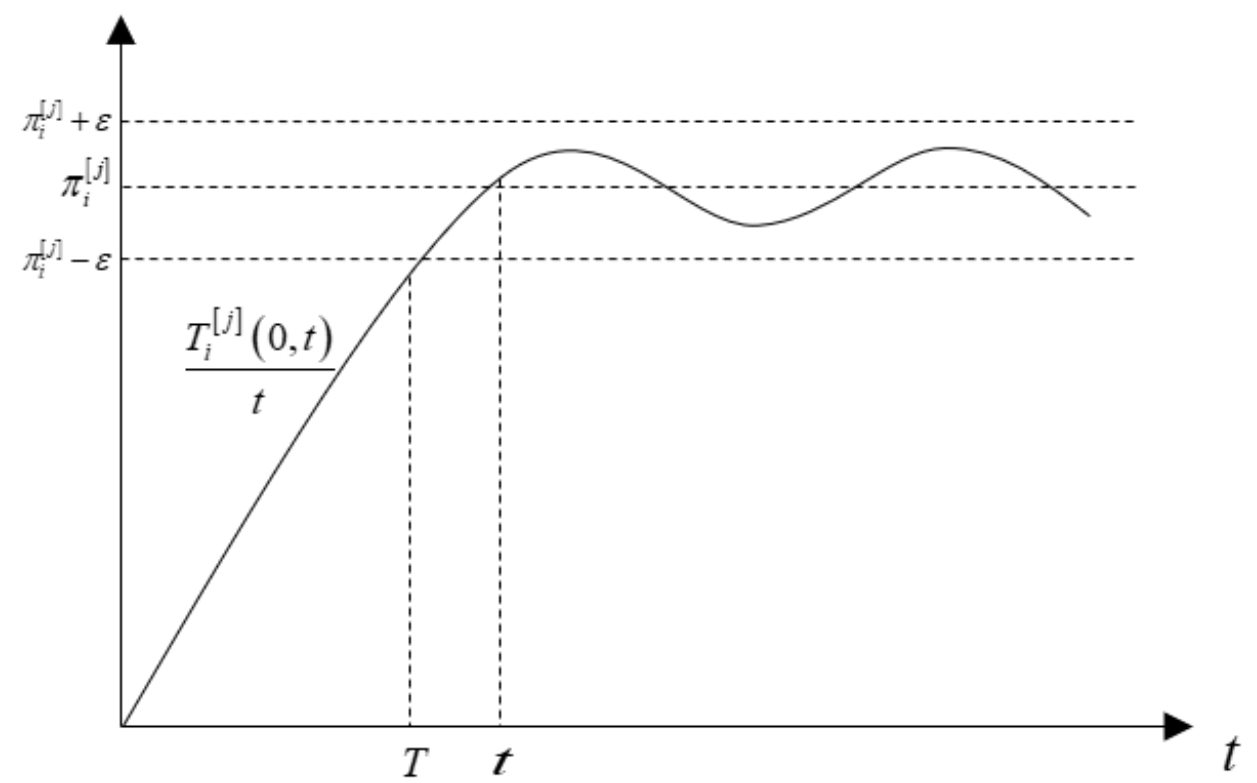

Figure 2. Schematic diagram.

Definition 1. (Ref. [2]) System (1) is characterized by the $\delta$-moment exponential stability if the following inequality is satisfied by positive real parameters of $\alpha$ and $\beta$

$$
\mathrm{E}\left[\|\boldsymbol{x}(t)\|^{\delta}\right]<\alpha e^{-\beta t}\|\boldsymbol{x}(0)\|^{\delta}
$$

when $x(0)$ and $\Pi(0)$ represent all positive initial conditions and all initial probability distributions.

When $\delta=1$ and $\delta=2$, the above definition is compatible with the well-known notion of 1-moment exponential stability and exponential mean-square stability (EMS stability), respectively.

Definition 2. (Ref. [4]) For any deterministic switching signal $\gamma_{k} \neq \gamma_{k+1}$ and all $0 \leq t \leq T$, the number of the deterministic switching signals occurring in the interval $[t, T]$ is denoted by $\phi(t, T)$ 
and the ADT of the deterministic switching signal during $[t, T]$ is denoted by $\tau^{*}$. A positive number $N_{0}$, called the chatter bound, exists such that it satisfies

$$
\phi(t, T) \leq N_{0}+\frac{T-t}{\tau^{*}}, \forall 0 \leq t \leq T
$$

Theorem 2. Consider the DSLCTPS (1). Assume that strictly positive vectors exist such that $c_{i}^{[j]}>1, \xi^{[j]}>0, \lambda_{i}^{[j]}>0, \mu_{i}^{[j]}>0$, where $\ln \mu_{i}^{[j]}<\lambda_{i}^{[j]}, i, r, R \in \overline{\mathrm{N}}, j, g \in \overline{\mathrm{M}}$ such that the inequalities given below hold:

$$
\begin{gathered}
(H 1)\left(c_{r}^{[g]}\right)^{T} \leq \xi^{[j]}\left(c_{i}^{[j]}\right)^{T} ; \\
(H 2)\left(c_{i}^{[j]}\right)^{T} A_{i}^{[j]}+\lambda_{i}^{[j]}\left(c_{i}^{[j]}\right)^{T}<0 ; \\
(H 3)\left(c_{i}^{[j]}\right)^{T} \leq \mu_{i}^{[j]}\left(c_{R}^{[j]}\right)^{T} ;
\end{gathered}
$$

Now, system (1) is 1-moment exponentially stable when the ADT of the deterministic switching signal satisfies

$$
\begin{gathered}
(H 4) \frac{\bar{\xi}}{\tau^{*}}+\bar{w}<0 ; \\
\bar{w}=\max _{j \in \overline{\mathrm{M}}}\left\{\theta^{[j]}+\rho^{[j]}\right\}, \theta^{[j]}=\sum_{i=1}^{\mathrm{N}} \pi_{i}^{[j]}\left|q_{i i}^{[j]}\right| \times \ln \left(\mu_{i}^{[j]}\right), \rho^{[j]}=-\sum_{i=1}^{\mathrm{N}} \lambda_{i}^{[j]} \times \pi_{i}^{[j]}, \\
\bar{\xi}=\max _{j \in \overline{\mathrm{M}}}\left\{\ln \xi^{[j]}\right\}
\end{gathered}
$$

Proof. Refer to Appendix A.

Theorem 3. When the ADT of the deterministic switching signal satisfies (H4) of Theorem 2, and $\xi^{[j]}$ meets (H1), the system (1) meets 1-moment exponential stability if and only if $\operatorname{sgn} \bar{A}$ meets the Hurwitz stability:

$$
\begin{gathered}
\overline{\boldsymbol{A}}=\mathbf{N} \times \text { blockdiag }\left(\left(\boldsymbol{A}_{1}^{[j]}\right)^{T},\left(\boldsymbol{A}_{2}^{[j]}\right)^{T}, \ldots,\left(\boldsymbol{A}_{N}^{[j]}\right)^{T}\right)+\boldsymbol{\Lambda} \otimes \boldsymbol{I}_{n} \\
\boldsymbol{\Lambda}=\left[\begin{array}{cccc}
\lambda_{1}^{[j]} \mu_{1}^{[j]} & \lambda_{1}^{[j]} \mu_{1}^{[j]} & \ldots & \lambda_{1}^{[j]} \mu_{1}^{[j]} \\
\lambda_{2}^{[j]} \mu_{2}^{[j]} & \lambda_{2}^{[j]} \mu_{2}^{[j]} & \ldots & \lambda_{2}^{[j]} \mu_{2}^{[j]} \\
\vdots & \vdots & \ddots & \vdots \\
\lambda_{N}^{[j]} \mu_{N}^{[j]} & \lambda_{N}^{[j]} \mu_{N}^{[j]} & \ldots & \lambda_{N}^{[j]} \mu_{N}^{[j]}
\end{array}\right] .
\end{gathered}
$$

Proof. Let $\left(\boldsymbol{c}_{i}^{[j]}\right)^{T} \boldsymbol{A}_{i}^{[j]}+\lambda_{i}^{[j]} \mu_{i}^{[j]}\left(\boldsymbol{c}_{R}^{[j]}\right)^{T}<0, i, R \in \overline{\mathrm{N}}, j \in \overline{\mathrm{M}}$, because $\left(\boldsymbol{c}_{i}^{[j]}\right)^{T} \leq \mu_{i}^{[j]}\left(\boldsymbol{c}_{R}^{[j]}\right)^{T}$, which is derived from (H3) of Theorem 2 , then

$$
\left(\boldsymbol{c}_{i}^{[j]}\right)^{T} \boldsymbol{A}_{i}^{[j]}+\lambda_{i}^{[j]}\left(\boldsymbol{c}_{i}^{[j]}\right)^{T}<\left(\boldsymbol{c}_{i}^{[j]}\right)^{T} \boldsymbol{A}_{i}^{[j]}+\lambda_{i}^{[j]} \mu_{i}^{[j]}\left(\boldsymbol{c}_{R}^{[j]}\right)^{T}
$$

(9) allows (H2) of Theorem 2 to hold. Thus,

$$
\left(c_{i}^{[j]}\right)^{T} \boldsymbol{A}_{i}^{[j]}+\lambda_{i}^{[j]} \mu_{i}^{[j]}\left(\boldsymbol{c}_{R}^{[j]}\right)^{T}<0
$$

It can guarantee that (H2) and (H3) of Theorem 2 have solutions. If (10) holds, then

$$
\sum_{R=1}^{\mathrm{N}}\left[\left(\boldsymbol{c}_{i}^{[j]}\right)^{T} \boldsymbol{A}_{i}^{[j]}+\lambda_{i}^{[j]} \mu_{i}^{[j]}\left(\boldsymbol{c}_{R}^{[j]}\right)^{T}\right]=\left(\boldsymbol{c}_{i}^{[j]}\right)^{T}\left(\mathrm{~N} \boldsymbol{A}_{i}^{[j]}\right)+\lambda_{i}^{[j]} \mu_{i}^{[j]} \sum_{R=1}^{\mathrm{N}}\left(\boldsymbol{c}_{R}^{[j]}\right)^{T}<0
$$


when (11) is only a necessary condition. Let $\operatorname{sgn}\left(A_{i}^{[j]}\right)=\operatorname{sgn}\left(\mathrm{N} A_{i}^{[j]}\right)$, then

$$
\left(c_{i}^{[j]}\right)^{T}\left[\operatorname{sgn}\left(A_{i}^{[j]}\right)\right]+\left[\operatorname{sgn}\left(\lambda_{i}^{[j]} \mu_{i}^{[j]}\right)\right]\left(c_{R}^{[j]}\right)^{T}
$$

and

$$
\left(c_{i}^{[j]}\right)^{T}\left[\operatorname{sgn}\left(\mathrm{N} A_{i}^{[j]}\right)\right]+\left[\operatorname{sgn}\left(\lambda_{i}^{[j]} \mu_{i}^{[j]}\right)\right] \sum_{R=1}^{\mathrm{N}}\left(c_{R}^{[j]}\right)^{T}
$$

are sign equivalent.

Let $v_{c}=\operatorname{vec}\left(c_{R}^{[j]}\right)$. It is immediate to verify that the previous inequation in (11) can be rewritten as $\bar{A} \cdot v_{\mathcal{C}}<0$, since $\bar{A}$ is a Metzler matrix there is a strictly positive vector $v_{\mathcal{C}}$ that meets $\bar{A} \cdot v_{\mathcal{C}}<0$ if and only if $\bar{A}$ meets the Hurwitz stability.

Since (12) and (13) are sign equivalent, system (1) is 1-moment exponentially stable; that is, $\operatorname{sgn} \bar{A}$ meets the Hurwitz stability.

Theorem 4. Assume system (1). Assume that there are symmetrically positive definite matrices $\boldsymbol{P}_{i}^{[j]} \in \boldsymbol{R}_{n \times n}, \lambda_{i}^{[j]}>0, \mu_{i}^{[j]}>0, i \in \overline{\mathrm{N}}, j, R \in \overline{\mathrm{M}}$, which satisfy the following inequalities:

$$
\begin{aligned}
& (H 1)\left(\boldsymbol{A}_{i}^{[j]}\right)^{T} \boldsymbol{P}_{i}^{[j]}+\boldsymbol{P}_{i}^{[j]} \boldsymbol{A}_{i}^{[j]}+\lambda_{i}^{[j]} \boldsymbol{P}_{i}^{[j]}<0 ; \\
& (H 2) \boldsymbol{P}_{i}^{[j]} \leq \mu_{i}^{[j]} \boldsymbol{P}_{R}^{[j]} ; \\
& (H 3) \eta^{[j]}=\sum_{i=1}^{\mathrm{N}} \pi_{i}^{[j]}\left(\left|q_{i i}^{[j]}\right| \ln \left(\mu_{i}^{[j]}\right)-\lambda_{i}^{[j]}\right)<0 ;
\end{aligned}
$$

Now, system (1) is EMS stable under the upcoming switching mechanism

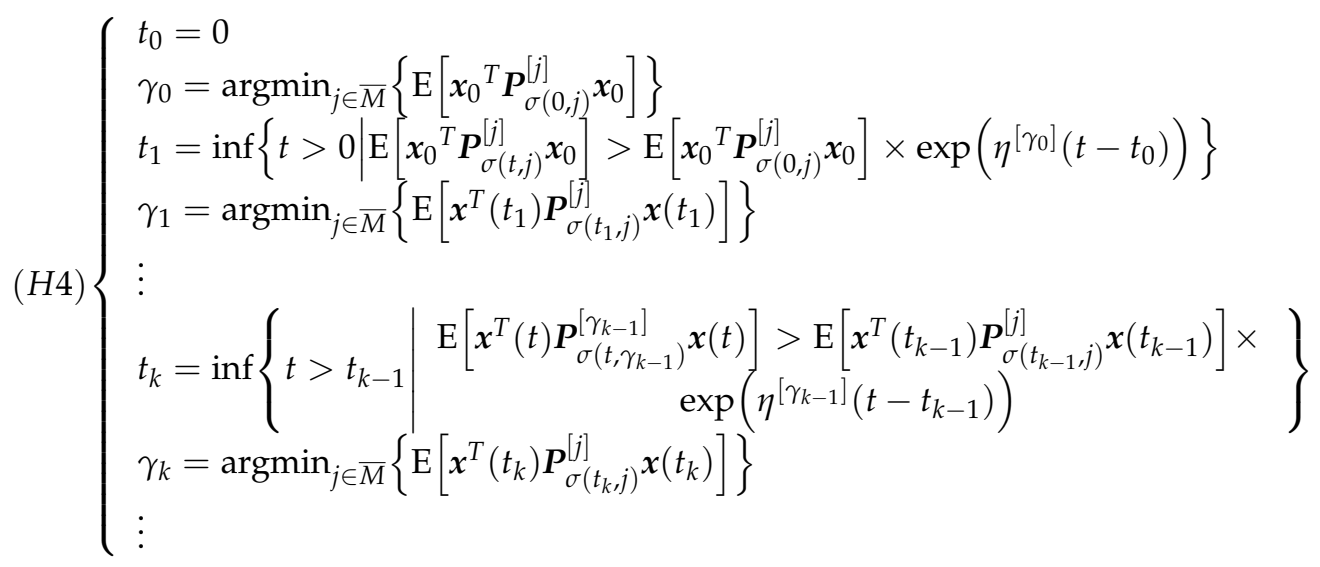

where the SS of the switching signal is determined deterministically, $\gamma(t)$ in $[0,+\infty)$ is denoted by $0=t_{0}<t_{1}<\ldots<t_{k}<\ldots$ as the switching subscript sequence of the switching signal determined deterministically and $\gamma(t)$ is denoted by $\left\{\gamma_{k} \in \overline{\mathrm{M}} \mid k=0,1,2, \ldots\right\}$

Proof. Refer to Appendix B.

Theorem 5. Consider the deterministic switching strategy (H4) of Theorem 4, while $\mu_{i}^{[j]}$ allows (H3) to hold, the system in (1) meets EMS stability if and only if sgñ $\tilde{A}$ is Hurwitz stable.

$$
\boldsymbol{\Lambda}=\left[\begin{array}{cccc}
\lambda_{1}^{[j]} \mu_{1}^{[j]} & \lambda_{1}^{[j]} \mu_{1}^{[j]} & \ldots & \lambda_{1}^{[j]} \mu_{1}^{[j]} \\
\lambda_{2}^{[j]} \mu_{2}^{j]} & \lambda_{2}^{[j]} \mu_{2}^{[j]} & \ldots & \lambda_{2}^{[j]} \mu_{2}^{[j]} \\
\vdots & \vdots & \ddots & \vdots \\
\lambda_{N}^{[j]} \mu_{N}^{[j]} & \lambda_{N}^{[j]} \mu_{N}^{[j]} & \ldots & \lambda_{N}^{[j]} \mu_{N}^{[j]}
\end{array}\right],
$$




$$
\tilde{\boldsymbol{A}}=\mathrm{N} \times \text { blockdiag }\left(\left(A_{1}^{[j]}\right)^{T} \oplus\left(\boldsymbol{A}_{1}^{[j]}\right)^{T},\left(\boldsymbol{A}_{2}^{[j]}\right)^{T} \oplus\left(\boldsymbol{A}_{2}^{[j]}\right)^{T}, \ldots,\left(\boldsymbol{A}_{\mathrm{N}}^{[j]}\right)^{T} \oplus\left(\boldsymbol{A}_{\mathrm{N}}^{[j]}\right)^{T}\right)+\boldsymbol{\Lambda} \otimes \boldsymbol{I}_{n^{2}} .
$$

Proof. Let

$$
\left(\boldsymbol{A}_{i}^{[j]}\right)^{T} \boldsymbol{P}_{i}^{[j]}+\boldsymbol{P}_{i}^{[j]} \boldsymbol{A}_{i}^{[j]}+\lambda_{i}^{[j]} \mu_{i}^{[j]} \boldsymbol{P}_{R}^{[j]}<0, \forall i, R \in \overline{\mathrm{N}}, \forall j \in \overline{\mathrm{M}}
$$

Considering $(\mathrm{H} 2)$ of Theorem 4 , then

$$
\left(\boldsymbol{A}_{i}^{[j]}\right)^{T} \boldsymbol{P}_{i}^{[j]}+\boldsymbol{P}_{i}^{[j]} \boldsymbol{A}_{i}^{[j]}+\lambda_{i}^{[j]} \boldsymbol{P}_{i}^{[j]}<\left(\boldsymbol{A}_{i}^{[j]}\right)^{T} \boldsymbol{P}_{i}^{[j]}+\boldsymbol{P}_{i}^{[j]} \boldsymbol{A}_{i}^{[j]}+\lambda_{i}^{[j]} \mu_{i}^{[j]} \boldsymbol{P}_{R}^{[j]}<0
$$

Satisfies (H1) of Theorem 4. From (14), then

$$
\begin{gathered}
\sum_{R=1}^{\mathrm{N}}\left[\left(\boldsymbol{A}_{i}^{[j]}\right)^{T} \boldsymbol{P}_{i}^{[j]}+\boldsymbol{P}_{i}^{[j]} \boldsymbol{A}_{i}^{[j]}+\lambda_{i}^{[j]} \mu_{i}^{[j]} \boldsymbol{P}_{R}^{[j]}\right]= \\
\left(\mathrm{N} \boldsymbol{A}_{i}^{[j]}\right)^{T} \boldsymbol{P}_{i}^{[j]}+\boldsymbol{P}_{i}^{[j]}\left(\mathrm{N} \boldsymbol{A}_{i}^{[j]}\right)+\lambda_{i}^{[j]} \mu_{i}^{[j]} \sum_{R=1}^{\mathrm{N}} \boldsymbol{P}_{R}^{[j]}<0
\end{gathered}
$$

The inequation in (16) proves the necessary condition. $\operatorname{As} \operatorname{sgn}\left(A_{i}^{[j]}\right)=\operatorname{sgn}\left(\mathrm{N} A_{i}^{[j]}\right)$,

$$
\operatorname{sgn}\left[\left(\boldsymbol{A}_{i}^{[j]}\right)^{T}\right] \boldsymbol{P}_{i}^{[j]}+\boldsymbol{P}_{i}^{[j]} \operatorname{sgn}\left(\boldsymbol{A}_{i}^{[j]}\right)+\operatorname{sgn}\left(\lambda_{i}^{[j]} \mu_{i}^{[j]}\right) \boldsymbol{P}_{R}^{[j]}
$$

and

$$
\operatorname{sgn}\left[\left(\mathrm{N} \boldsymbol{A}_{i}^{[j]}\right)^{T}\right] \boldsymbol{P}_{i}^{[j]}+\boldsymbol{P}_{i}^{[j]} \operatorname{sgn}\left(\mathrm{N} \boldsymbol{A}_{i}^{[j]}\right)+\operatorname{sgn}\left(\lambda_{i}^{[j]} \mu_{i}^{[j]}\right) \sum_{R=1}^{\mathrm{N}} \boldsymbol{P}_{R}^{[j]}
$$

are sign equivalent. Using $\operatorname{vec}(\boldsymbol{A X} \boldsymbol{B})=\left(\boldsymbol{B}^{T} \otimes \boldsymbol{A}\right) \operatorname{vec}(\boldsymbol{X})$, from (16), we obtain

$$
\begin{aligned}
& {\left[\boldsymbol{I} \otimes\left(\mathbf{N} \boldsymbol{A}_{i}^{[j]}\right)^{T}\right] \operatorname{vec}\left(\boldsymbol{P}_{i}^{[j]}\right)+\left[\left(\mathbf{N} \boldsymbol{A}_{i}^{[j]}\right)^{T} \otimes \boldsymbol{I}\right] \operatorname{vec}\left(\boldsymbol{P}_{i}^{[j]}\right)+\sum_{R=1}^{\mathrm{N}}\left(\lambda_{i}^{[j]} \mu_{i}^{[j]}\right) \operatorname{vec}\left(\boldsymbol{P}_{R}^{[j]}\right)} \\
& =\left[\left(\mathbf{N} \boldsymbol{A}_{i}^{[j]}\right)^{T} \oplus\left(\mathrm{N} \boldsymbol{A}_{i}^{[j]}\right)^{T}\right] \operatorname{vec}\left(\boldsymbol{P}_{i}^{[j]}\right)+\sum_{R=1}^{\mathrm{N}}\left(\lambda_{i}^{[j]} \mu_{i}^{[j]}\right) \operatorname{vec}\left(\boldsymbol{P}_{R}^{[j]}\right)<0
\end{aligned}
$$

Hence, (19) can be expressed as

$$
\tilde{\boldsymbol{A}}\left(\begin{array}{c}
\operatorname{vec} \boldsymbol{P}_{1}^{[j]} \\
\operatorname{vec} \boldsymbol{P}_{2}^{[j]} \\
\vdots \\
\operatorname{vec} \boldsymbol{P}_{N}^{[j]}
\end{array}\right)<0
$$

if there are symmetrically positive definite matrices $P_{i}^{[j]}$, equivalently, if and only if $\tilde{A}$ has the Hurwitz stability property.

Since (17) and (18) are sign equivalent, the system in (1) is EMS stable, equivalently, if and only if $\operatorname{sgn} \tilde{A}$ is Hurwitz stable.

\subsection{Qualitative Characterization of Dual Switching Linear Continuous-Time Positive Systems}

The assumption in this research is that the numerical realization of $A_{i}^{[j]}$ and $\boldsymbol{\Lambda}$ are not known. $A_{i}^{[j]}$ and $\Lambda$ belong to a class of structured matrices whose elements contain given signs. A matrix with signs is defined where all elements are represented by signs belonging to $S=\{0,-,+\}$. The set of sign matrices whose orders are $n$ by $m$ is denoted by $S_{n \times m}$. Matrices consisting of real numbers with specific classifications agree upon are inherently 
compatibility with the above matrices where their features are not related to the elements that have special values.

A corresponding unique sign matrix can be constructed by allocating a member of the set $S=\{0,-,+\}$ to its elements $A_{i, j}$ based on its sign for a given matrix $A$. Due to having a similar sign matrix assigned by two different real matrices, the class of whole matrices consisting of real numbers corresponding to a sign matrix can be defined as follows.

Definition 3. (Ref. [20]) The qualitative group of the matrix A consisting of real numbers whose order in $n$ by $m$ can be defined by

$$
Q(A)=\left\{\boldsymbol{B} \in \boldsymbol{R}_{n \times m} \mid \operatorname{sgn} \boldsymbol{B}_{i, j}=\operatorname{sgn} \boldsymbol{A}_{i, j}, i=1, \ldots n, j=1, \ldots, m\right\} .
$$

A unique qualitative group for each sign matrix can be observed. Suppose that $Q_{S}$ designates the qualitative class $Q(A)$, where $\operatorname{sgn} A_{i, j}=S_{i, j}$ holds for some matrix $A$ consisting of real numbers with proper dimension for a certain sign matrix $S$. Correspondingly $\lambda_{i}^{[j]}>0, \mu_{i}^{[j]}>0$ and $Q_{\Lambda}=Q_{S} \cap\{+\}$ for a sign matrix $\Lambda$ whose order is $N$ by N. Note that if $S_{i, j} \in\{-, 0\}$, then $Q_{\Lambda}$ is an empty set. Table 1 presents the definitions of the aforementioned sets.

Table 1. Set reference list.

\begin{tabular}{ll}
\hline Symbol & Set Definition \\
\hline $\operatorname{sgn} A_{n \times m}$ & $A$ is an n-by-m matrix with elements belonging to $\{0,-,+\}$. \\
$Q(A)$ & The qualitative class associated with the real. \\
$Q_{S}$ & $Q(A)$ s.t. $\operatorname{sgn} A_{i, j}=S_{i, j}$. \\
$Q_{\boldsymbol{\Lambda}}$ & $Q_{\boldsymbol{\Lambda}}=Q_{S} \cap\{+\}$. \\
\hline
\end{tabular}

Subsystem matrices whose given structures are known can be expressed by corresponding sign matrices. Therefore, the definition of the system denoted by (1) is expressed as follows:

$$
\left\{\begin{array}{l}
\dot{x}(t)=A_{\sigma(t, \gamma(t))}^{[\gamma(t)]} x(t), t>0 \\
A_{i}^{[j]} \in Q_{S_{i}}, \forall i \in \overline{\mathrm{N}}, j \in \overline{\mathrm{M}} \\
\boldsymbol{\Lambda} \in Q_{\boldsymbol{\Lambda}}
\end{array}\right.
$$

In the above relation, all $A_{i}^{[j]}, \lambda_{i}^{[j]}, \mu_{i}^{[j]}(\forall i \in \overline{\mathrm{N}}, j \in \overline{\mathrm{M}})$ are unknown but belong to the pre-defined qualitative classes $Q_{S_{i}}$ and $Q_{\Lambda}$.

Definition 4. (Ref. [26]) If there is $A_{i}^{[j]}(\forall i \in \overline{\mathrm{N}}, j \in \overline{\mathrm{M}})$ in $Q_{s_{i}}$ and $\boldsymbol{\Lambda}$ in $Q_{\boldsymbol{\Lambda}}$ such that system (1) is 1-moment exponentially stable or EMS stable, then (21) is called 1-moment exponentially sign stable or EMS sign stable.

\section{Sign Stability Concept}

A variety of subsystem prototypes defined by sign matrices $S_{i}$, to express various qualitative classes, is admitted by System (21). $S$ should be generalized to tackle the ambiguity led by sign matrices $S_{i}$ with various nonzero elements. Therefore, some subsystem structures in (21) can be embedded into a single matrix.

Definition 5. (Ref. [21]) The matrix $\boldsymbol{A}$ is sign stable if every matrix $\boldsymbol{B}$ with a similar sign pattern to $A\left(\operatorname{sgn} b_{i j}=\operatorname{sgn} a_{i j}, \forall i, j\right)$ is Hurwitz stable, regardless of the values of $b_{i j}$.

Definition 6. If $\operatorname{sgn}\left[A=\left(a_{i j}\right)_{\mathrm{n} \times n}\right]$ satisfies $a_{i j} a_{j k} \ldots a_{q r} a_{r i}=0$ for all sequences with two or more different indices $i, j, k, \ldots, q, r$, then $G_{A}$ is called acyclic. 
Theorem 6. (Ref. [20]) All of these declarations are equivalent:

(i) $G_{A}$ is acyclic;

(ii) There is a permutation matrix $\boldsymbol{P}$ where $\boldsymbol{P}^{T} \boldsymbol{A P}$ is upper triangular.

Definition 7. (Ref. [21]) The joining operation of each sign pattern can be defined to establish a particular sign pattern called the initial sign pattern of the set based on the isogenous term set, the mentioned steps satisfy the conditions below.

(1) For a specific location of any sign pattern whose elements are denoted by 0 or the nonzero sign, the corresponding ones in the original sign pattern can be composed of a similar nonzero sign.

(2) For a specific location of any sign pattern whose elements are all equal to 0 , the corresponding one in the original sign pattern is also 0.

(3) For a specific location of any sign pattern whose elements consist of distinct signs, the corresponding ones in the initial sign pattern should be employed to define all possible signs, "*".

$$
\left\{\left(S_{i}\right)_{k, l},\left(S_{j}\right)_{k, l}\right\} \rightarrow\left\{\begin{array}{l}
-,\left\{\left(S_{i}\right)_{k, l}\left(s_{j}\right)_{k, l}\right\} \subseteq\{-, 0\},\left\{\left(S_{i}\right)_{k, l}\left(s_{j}\right)_{k, l}\right\} \not \subset\{0\} \\
0,\left(S_{i}\right)_{k, l}=\left(S_{j}\right)_{k, l}=0 \\
+,\left\{\left(S_{i}\right)_{k, l}\left(S_{j}\right)_{k, l}\right\} \subseteq\{+, 0\},\left\{\left(S_{i}\right)_{k, l},\left(S_{j}\right)_{k, l}\right\} \not \subset\{0\} \\
*, \text { otherwise }
\end{array}\right.
$$

A bounded set of sign matrices $S$ is considered, the original sign pattern is expressed as $\boldsymbol{R}=\sum_{S_{i} \in S} S_{i}$.

For example, if

$$
\left\{\boldsymbol{S}_{1}, S_{2}, S_{3}\right\}=\left\{\left[\begin{array}{ccc}
- & + & + \\
- & 0 & - \\
0 & + & -
\end{array}\right],\left[\begin{array}{ccc}
- & 0 & + \\
- & - & - \\
0 & 0 & -
\end{array}\right],\left[\begin{array}{ccc}
0 & + & - \\
- & - & 0 \\
0 & + & -
\end{array}\right]\right\},
$$

then, its original sign pattern is

$$
\boldsymbol{R}=\left[\begin{array}{ccc}
- & + & * \\
- & - & - \\
0 & + & -
\end{array}\right]
$$

Theorem 7. (Gershgorin's Circle Theorem)

Each eigenvalue of the $n$-order matrix $A$ is located in the following $n$ circles

$$
C_{i}:\left\{\lambda|| \lambda-a_{i i}\left|\leq \sum_{j \neq i, j=1}^{n}\right| a_{i j} \mid\right\}, i=1,2, \ldots, n
$$

Gershgorin's Circle Theorem used in this paper is a relatively conservative theorem. In the analysis of sign stability the boundary of the circle is not discussed, only the eigenvalues contained in the circle are analyzed. The problem of the boundary of the disc needs further discussion.

Theorem 8. (Ref. [18]) Assume that $A$ could be a partitioned matrix whose partitions are denoted by $\boldsymbol{A}_{i}(i=1,2, \ldots, n)$, with the property that $\operatorname{det} A=\operatorname{det} \boldsymbol{A}_{1} \times \operatorname{det} \boldsymbol{A}_{2} \times \ldots \operatorname{det} \boldsymbol{A}_{n}$, then $A$ is called sign stable, if and only if each partition matrix $A_{i}$ is sign stable. 


\section{1-Moment Exponential Sign Stability and EMS Sign Stability}

The sign matrices $S_{i}$ and $\boldsymbol{\Lambda}$ of the DSLCTS (21) are considered. The representative matrix $S=\left\{S_{1}, \ldots, S_{N}\right\}$ is denoted by $R$ and $\bar{S}$ is expressed by

$$
\overline{\boldsymbol{S}}=\left[\begin{array}{cccc}
\left(\boldsymbol{S}_{1}\right)^{T}+\boldsymbol{\Lambda}_{1,1} \cdot \boldsymbol{I}_{n} & \boldsymbol{\Lambda}_{1,2} \cdot \boldsymbol{I}_{n} & \ldots & \boldsymbol{\Lambda}_{1, N} \cdot \boldsymbol{I}_{n} \\
\boldsymbol{\Lambda}_{2,1} \cdot \boldsymbol{I}_{n} & \left(\boldsymbol{S}_{2}\right)^{T}+\boldsymbol{\Lambda}_{2,2} \cdot \boldsymbol{I}_{n} & \ldots & \boldsymbol{\Lambda}_{2, N} \cdot \boldsymbol{I}_{n} \\
\vdots & \vdots & \ddots & \vdots \\
\boldsymbol{\Lambda}_{N, 1} \cdot \boldsymbol{I}_{n} & \boldsymbol{\Lambda}_{N, 2} \cdot \boldsymbol{I}_{n} & \ldots & \left(\boldsymbol{S}_{N}\right)^{T}+\boldsymbol{\Lambda}_{N, N} \cdot \boldsymbol{I}_{n}
\end{array}\right]
$$

where $\bar{S}$ denotes the expanded parallel sign matrix $\bar{A}$ consisting of real numbers, where its graph reflects how the graph $\boldsymbol{\Lambda}$ interconnects and meshes with particular subsystems when the DSLCTS is a concern.

To clarify the mentioned interaction, assume (21) with

$$
S_{1}=\left[\begin{array}{cc}
- & + \\
0 & -
\end{array}\right], S_{2}=\left[\begin{array}{cc}
- & 0 \\
+ & -
\end{array}\right], \Lambda=\left[\begin{array}{cc}
+ & + \\
+ & +
\end{array}\right]
$$

then $\bar{S}$ can take the form given below utilizing the case above

$$
\overline{\boldsymbol{S}}=\left[\begin{array}{cccc}
* & 0 & + & 0 \\
+ & * & 0 & + \\
+ & 0 & * & + \\
0 & + & 0 & *
\end{array}\right]
$$

Figure 3 depicts $\bar{S}^{\prime}$ s directed graph. In $G_{\bar{S}}$, the graph $\Lambda$ adjoins the above two acyclic graphs. The sign stability $\bar{S}$ is verified by analyzing the relationship of $S_{1}, S_{2}$, and $\Lambda$.

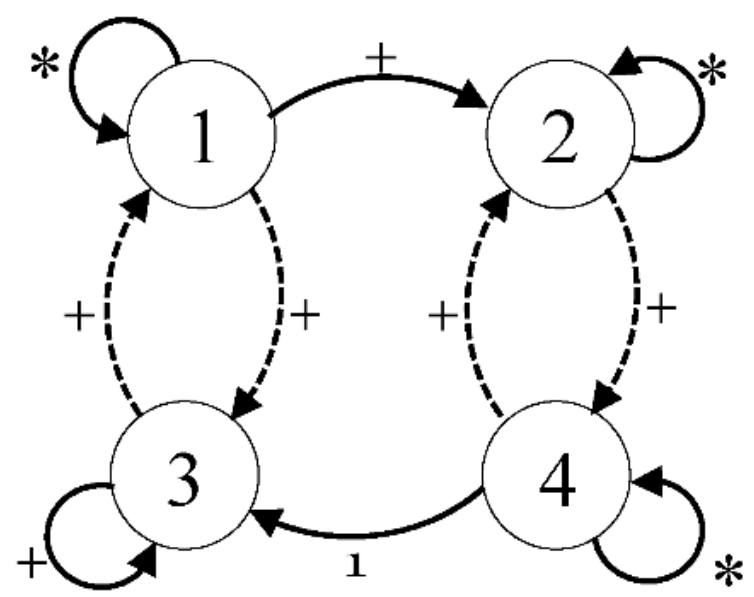

Figure 3. $\overline{S^{\prime}}$ s directed graph.

\subsection{1-Moment Exponential Sign Stability Analysis}

Theorem 9. Assume that the class of sign matrices is represented by $\boldsymbol{R} . \boldsymbol{S}_{i}=\operatorname{sgn} \boldsymbol{A}_{i}^{[j]}$. The representations below are mathematically equal:

(i) $\operatorname{sgn} \bar{A}$ is sign stable;

(ii) $G_{\boldsymbol{R}}$ is acyclic, $\operatorname{diag}\left[\boldsymbol{A}_{i}^{[j]}+\lambda_{i}^{[j]} \mu_{i}^{[j]} \mathbf{I}_{n}\right]<0$.

Proof. Consider that $\boldsymbol{P}=\left[\boldsymbol{P}_{1}, \boldsymbol{P}_{2}, \ldots, \boldsymbol{P}_{N}\right]$ is an $n \mathrm{~N} \times n \mathrm{~N}$ permutation matrix, where $\boldsymbol{P}_{i}$ is an $n \mathrm{~N} \times \mathrm{N}$ matrix with the canonical column vectors $\boldsymbol{e}_{v}$ as its columns; that is, 


$$
\begin{gathered}
\boldsymbol{P}_{i}=\left[\boldsymbol{e}_{v_{1}^{i}}, \boldsymbol{e}_{v_{2}^{i}}, \ldots, \boldsymbol{e}_{v_{\mathrm{N}}^{i}}\right] \text { and }\left\{v_{1}^{i}, v_{2}^{i}, \ldots, v_{\mathrm{N}}^{i}\right\} \text { such that } v_{h}^{i}=i+(h-1) n, \\
\boldsymbol{D}_{i, v}=\text { blockdiag }\left(\left(\boldsymbol{S}_{1}\right)_{i, v} \ldots,\left(\boldsymbol{S}_{\mathrm{N}}\right)_{i, v}\right), \boldsymbol{S}_{i}=\operatorname{sgn} \boldsymbol{A}_{i}^{[j]}=\operatorname{sgn}\left(\mathbf{N} \boldsymbol{A}_{i}^{[j]}\right), \\
\boldsymbol{P}^{T}(\operatorname{sgn} \overline{\boldsymbol{A}}) \boldsymbol{P}=\left[\begin{array}{cccc}
\boldsymbol{\Lambda}+\boldsymbol{D}_{1,1} & \boldsymbol{D}_{2,1} & \ldots & \boldsymbol{D}_{n, 1} \\
\boldsymbol{D}_{1,2} & \boldsymbol{\Lambda}+\boldsymbol{D}_{2,2} & \ldots & \boldsymbol{D}_{n, 2} \\
\vdots & \vdots & \ddots & \vdots \\
\boldsymbol{D}_{1, n} & \boldsymbol{D}_{2, n} & \ldots & \boldsymbol{\Lambda}+\boldsymbol{D}_{n, n}
\end{array}\right]
\end{gathered}
$$

As $G_{S_{i}}$ is acyclic, $G_{R}$ is acyclic by Definition 7. Therefore, a permutation matrix $Q$ exists where $Q^{T} S_{i} Q$ is upper triangular by Theorem 6 and one of $\boldsymbol{D}_{i, v}$ and $\boldsymbol{D}_{v, i}$ are zero matrices. Since $G_{P^{T}(\operatorname{sgn} \bar{A}) P}$ inherits graphical properties $G_{S_{i}}$ is a permutation matrix $Q^{\prime}$ such that $\boldsymbol{Q}^{\prime T}\left[\boldsymbol{P}^{T}(\operatorname{sgn} \bar{A}) \boldsymbol{P}\right] \boldsymbol{Q}^{\prime}$ is triangular matrix whose entities are located at the upper block and its block-diagonal matrices were $\boldsymbol{\Lambda}+\boldsymbol{D}_{k_{i} k_{i}}\left(k_{i}=1,2, \ldots, n\right)$. The process is as follows.

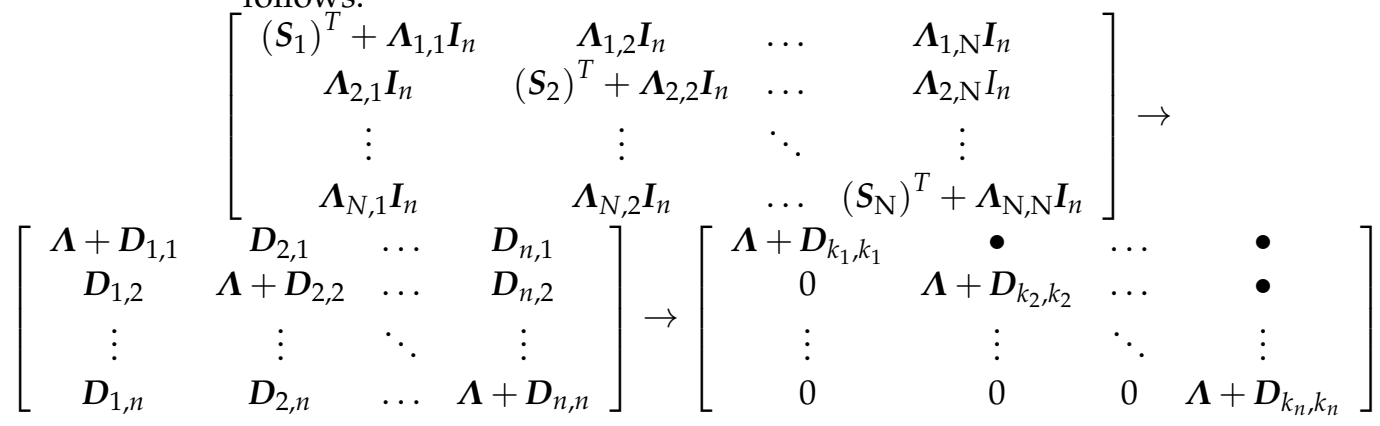

0 is a matrix where all of its elements are $0, \bullet$ is $\boldsymbol{D}_{i, v}$ or $\boldsymbol{D}_{v, i}$, which is not a matrix with all zero elements. If $\operatorname{sgn} \overline{\boldsymbol{A}}$ is sign stable, equivalently, $\boldsymbol{P}^{T}(\operatorname{sgn} \overline{\boldsymbol{A}}) \boldsymbol{P}$ is sign stable, equivalently, $\boldsymbol{Q}^{\prime T}\left[\boldsymbol{P}^{T}(\operatorname{sgn} \overline{\boldsymbol{A}}) \boldsymbol{P}\right] \boldsymbol{Q}^{\prime}$ is sign stable. Moreover, $\boldsymbol{Q}^{\prime T}\left[\boldsymbol{P}^{T}(\operatorname{sgn} \overline{\boldsymbol{A}}) \boldsymbol{P}\right] \boldsymbol{Q}^{\prime}$ is sign stable if and only if all $\boldsymbol{\Lambda}+\boldsymbol{D}_{k_{i}, k_{i}}$ are sign stable by Theorem 8 .

Since $S_{i}=\operatorname{sgn}\left(\mathrm{N} A_{i}^{[j]}\right)$, then

$$
\boldsymbol{\Lambda}+\boldsymbol{D}_{k_{i}, k_{i}}=\left[\begin{array}{cccc}
\mathrm{N}\left(\boldsymbol{A}_{1}^{[j]}\right)_{k_{i}, k_{i}}+\lambda_{1}^{[j]} \mu_{1}^{[j]} & \lambda_{1}^{[j]} \mu_{1}^{[j]} & \ldots & \lambda_{1}^{[j]} \mu_{1}^{[j]} \\
\lambda_{2}^{[j]} \mu_{2}^{[j]} & \mathrm{N}\left(A_{2}^{[j]}\right)_{k_{i}, k_{i}}+\lambda_{2}^{[j]} \mu_{2}^{[j]} & \ldots & \lambda_{2}^{[j]} \mu_{2}^{[j]} \\
\vdots & \vdots & \ddots & \vdots \\
\lambda_{\mathrm{N}}^{[j]} \mu_{\mathrm{N}}^{[j]} & \lambda_{\mathrm{N}}^{[j]} \mu_{\mathrm{N}}^{[j]} & \ldots & \mathrm{N}\left(A_{\mathrm{N}}^{[j]}\right)_{k_{i}, k_{i}}+\lambda_{\mathrm{N}}^{[j]} \mu_{\mathrm{N}}^{[j]}
\end{array}\right]
$$

Because $\operatorname{diag}\left[\boldsymbol{A}_{i}^{[j]}+\lambda_{i}^{[j]} \mu_{i}^{[j]} \boldsymbol{I}_{n}\right]<0$ and $\lambda_{i}^{[j]} \mu_{i}^{[j]}>0, \operatorname{diag}\left[\mathrm{N} \boldsymbol{A}_{i}^{[j]}+\mathrm{N} \lambda_{i}^{[j]} \mu_{i}^{[j]} \boldsymbol{I}_{n}\right]<0$, $\operatorname{diag}\left[\mathrm{N} \boldsymbol{A}_{i}^{[j]}+\left(\lambda_{i}^{[j]} \mu_{i}^{[j]}\right) \boldsymbol{I}_{n}\right]<-(\mathrm{N}-1) \lambda_{i}^{[j]} \mu_{i}^{[j]}$. Now, according to Theorem 7 and Definition $5, t_{i}^{[j]}=\left|\operatorname{diag}\left[\mathrm{N} A_{i}^{[j]}+\left(\lambda_{i}^{[j]} \mu_{i}^{[j]}\right) \boldsymbol{I}_{n}\right]\right|, u_{i}^{[j]}=(\mathrm{N}-1) \lambda_{i}^{[j]} \mu_{i}^{[j]}$ as shown in Figure 4. Thus, $\boldsymbol{\Lambda}+\boldsymbol{D}_{k_{i}, k_{i}}$ are sign stable, leading to sign stability of $\operatorname{sgn} \overline{\boldsymbol{A}}$. 


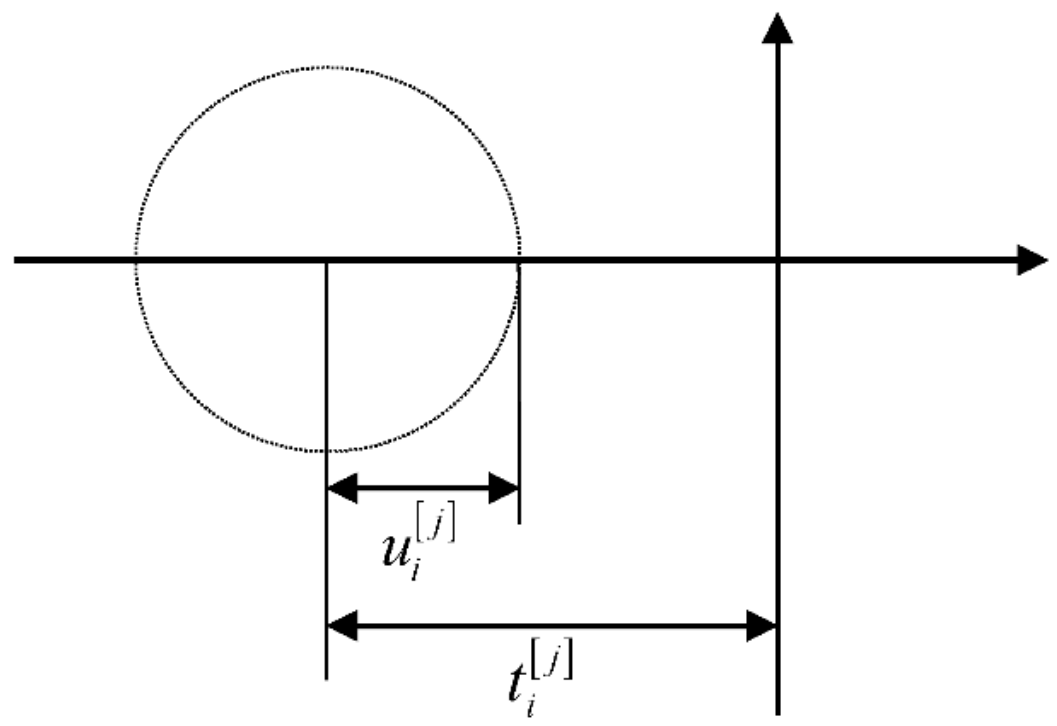

Figure 4. Distribution of eigenvalues.

Theorem 10. When the ADT of the deterministic switching signal satisfies (H4) of Theorem 2, and $\xi^{[j]}$ allows (H1) to hold, if for each $\forall i \in \overline{\mathrm{N}}, \forall j \in \overline{\mathrm{M}}$, $\operatorname{sgn} A_{i}^{[j]}$ satisfies Theorem 9, then the system (21) is 1-moment exponentially sign stable.

Proof. When the ADT of the deterministic switching signal satisfies (H4) of Theorem 2, and $\xi^{[j]}$ makes (H1) hold, system (1) meets 1-moment exponential stability if and only if $\operatorname{sgn} \bar{A}$ is Hurwitz stable by Theorem 3. According to Definition 4, if system (1) is 1-moment exponentially stable, and every $A_{i}^{[j]}(\forall i \in \overline{\mathrm{N}}, j \in \overline{\mathrm{M}})$ belongs to $Q_{s_{i}}$ and $\boldsymbol{\Lambda}$ belongs to $Q_{\Lambda}$, then (21) is 1-moment exponentially sign stable. Thus, every $A_{i}^{[j]}(\forall i \in \overline{\mathrm{N}}, j \in \overline{\mathrm{M}})$ belongs to $Q_{S_{i}}$ and $\Lambda$ belongs to $Q_{\Lambda}$, such that sgn $\bar{A}$ is sign stable, leading to 1-moment exponential sign stability of (21). Then, for each $\forall i \in \overline{\mathrm{N}}, \forall j \in \overline{\mathrm{M}}, \operatorname{sgn} A_{i}^{[j]}$ satisfies Theorem 9 , and the system (21) is 1-moment exponentially sign stable.

\subsection{EMS Sign Stability Analysis}

Theorem 11. Assume that the set of sign matrices $S_{i}=\operatorname{sgn} A_{i}^{[j]}$ are represented by $\boldsymbol{R}$. The representations below are mathematically equal:

(i) $\operatorname{sgn} \tilde{A}$ is sign stable;

(ii) $G_{\boldsymbol{R}}$ is acyclic, diag $\left[\boldsymbol{A}_{i}^{[j]}+\frac{1}{2} \lambda_{i}^{[j]} \mu_{i}^{[j]} \boldsymbol{I}_{n}\right]<0$.

Proof. Since $G_{\boldsymbol{R}}$ is acyclic, $G_{S_{i}}$ is also acyclic by Definition 7 ; that is, $S_{i}=\operatorname{sgn} A_{i}^{[j]}=\operatorname{sgn}\left(\mathrm{N} A_{i}^{[j]}\right)$,

$$
\left(\boldsymbol{S}_{i}\right)^{T} \oplus\left(\boldsymbol{S}_{i}\right)^{T}=\left[\begin{array}{cccc}
\left(\boldsymbol{S}_{i}\right)^{T}+\left(\boldsymbol{S}_{i}\right)_{1,1} \boldsymbol{I}_{n} & \left(\boldsymbol{S}_{i}\right)_{2,1} \boldsymbol{I}_{n} & \ldots & \left(\boldsymbol{S}_{i}\right)_{n, 1} \boldsymbol{I}_{n} \\
\left(\boldsymbol{S}_{i}\right)_{1,2} \boldsymbol{I}_{n} & \left(\boldsymbol{S}_{i}\right)^{T}+\left(\boldsymbol{S}_{i}\right)_{2,2} \boldsymbol{I}_{n} & \ldots & \left(\boldsymbol{S}_{i}\right)_{n, 2} \boldsymbol{I}_{n} \\
\vdots & \vdots & \ddots & \vdots \\
\left(\boldsymbol{S}_{i}\right)_{1, n} \boldsymbol{I}_{n} & \left(\boldsymbol{S}_{i}\right)_{2, n} \boldsymbol{I}_{n} & \ldots & \left(\boldsymbol{S}_{i}\right)^{T}+\left(\boldsymbol{S}_{i}\right)_{n, n} \boldsymbol{I}_{n}
\end{array}\right] .
$$


Thus, $G_{\left(S_{i}\right)^{T} \oplus\left(S_{i}\right)^{T}}$ inherits the graphical properties of $G_{S_{i}}, G_{\left(S_{i}\right)^{T} \oplus\left(S_{i}\right)^{T}}$ is acyclic, and $\tilde{S}$ stands for the extended sign matrix analogue of the real matrix $\tilde{A}$

$$
\tilde{\boldsymbol{S}}=\left[\begin{array}{cccc}
\left(\boldsymbol{S}_{1}\right)^{T} \oplus\left(\boldsymbol{S}_{1}\right)^{T}+\boldsymbol{\Lambda}_{1,1} \boldsymbol{I}_{n^{2}} & \boldsymbol{\Lambda}_{1,2} \boldsymbol{I}_{n^{2}} & \ldots & \boldsymbol{\Lambda}_{1, \mathrm{~N}} \boldsymbol{I}_{n^{2}} \\
\boldsymbol{\Lambda}_{2,1} \boldsymbol{I}_{n^{2}} & \left(\boldsymbol{S}_{2}\right)^{T} \oplus\left(\boldsymbol{S}_{2}\right)^{T}+\boldsymbol{\Lambda}_{2,2} \boldsymbol{I}_{n^{2}} & \ldots & \boldsymbol{\Lambda}_{2, \mathrm{~N}} \boldsymbol{I}_{n^{2}} \\
\vdots & \vdots & \ddots & \vdots \\
\boldsymbol{\Lambda}_{N, 1} \boldsymbol{I}_{n^{2}} & \boldsymbol{\Lambda}_{\mathrm{N}, 2} \boldsymbol{I}_{n^{2}} & \ldots & \left(\boldsymbol{S}_{\mathrm{N}}\right)^{T} \oplus\left(\boldsymbol{S}_{\mathrm{N}}\right)^{T}+\boldsymbol{\Lambda}_{\mathrm{N}, \mathrm{N}} \boldsymbol{I}_{n^{2}}
\end{array}\right]
$$

$G_{\tilde{S}}$ inherits the graphical properties of $G_{\bar{S}}$, and $\boldsymbol{D}_{i, v}^{\prime}=$ blockdiag $\left(\left(\left(S_{1}\right)^{T} \oplus\left(S_{1}\right)^{T}\right)_{i, v}\right.$ $\left.\ldots,\left(\left(\boldsymbol{S}_{\mathrm{N}}\right)^{T} \oplus\left(\boldsymbol{S}_{\mathrm{N}}\right)^{T}\right)_{i, v}\right)$ and we only prove that $\boldsymbol{\Lambda}+\boldsymbol{D}_{k_{i}, k_{i}}^{\prime}$ is sign stable.

Because $S_{i}=\operatorname{sgn}\left(\mathrm{N} A_{i}^{[j]}\right)$, then

$$
\boldsymbol{\Lambda}+\boldsymbol{D}_{k_{i}, k_{i}}^{\prime}=\left[\begin{array}{cccc}
2 \mathrm{~N}\left(\boldsymbol{A}_{1}^{[j]}\right)_{k_{i}, k_{i}}+\lambda_{1}^{[j]} \mu_{1}^{[j]} & \lambda_{1}^{[j]} \mu_{1}^{[j]} & \ldots & \lambda_{1}^{[j]} \mu_{1}^{[j]} \\
\lambda_{2}^{[j]} \mu_{2}^{[j]} & 2 \mathrm{~N}\left(A_{2}^{[j]}\right)_{k_{i}, k_{i}}+\lambda_{2}^{[j]} \mu_{2}^{[j]} & \ldots & \lambda_{2}^{[j]} \mu_{2}^{[j]} \\
\vdots & \vdots & \ddots & \vdots \\
\lambda_{\mathrm{N}}^{[j]} \mu_{\mathrm{N}}^{[j]} & \lambda_{\mathrm{N}}^{[j]} \mu_{\mathrm{N}}^{[j]} & \ldots & 2 \mathrm{~N}\left(A_{\mathrm{N}}^{[j]}\right)_{k_{i}, k_{i}}+\lambda_{\mathrm{N}}^{[j]} \mu_{\mathrm{N}}^{[j]}
\end{array}\right]
$$

Since $\operatorname{diag}\left[\boldsymbol{A}_{i}^{[j]}+\frac{1}{2} \lambda_{i}^{[j]} \mu_{i}^{[j]} \boldsymbol{I}_{n}\right] \quad<\quad 0 \quad$ and $\lambda_{i}^{[j]} \mu_{i}^{[j]}>0$, $\operatorname{diag}\left[\mathrm{N} \boldsymbol{A}_{i}^{[j]}+\frac{1}{2} \mathrm{~N} \lambda_{i}^{[j]} \mu_{i}^{[j]} \boldsymbol{I}_{n}\right]<0, \operatorname{diag}\left[\mathrm{N}\left(\boldsymbol{A}_{i}^{[j]}\right)^{T} \oplus\left(\boldsymbol{A}_{i}^{[j]}\right)^{T}+\mathrm{N}\left(\lambda_{i}^{[j]} \mu_{i}^{[j]}\right) \boldsymbol{I}_{n^{2}}\right]<0$, and diag $\left[\mathrm{N}\left(\boldsymbol{A}_{i}^{[j]}\right)^{T} \oplus\left(\boldsymbol{A}_{i}^{[j]}\right)^{T}+\left(\lambda_{i}^{[j]} \mu_{i}^{[j]}\right) \boldsymbol{I}_{n^{2}}\right]<-(\mathrm{N}-1)\left(\lambda_{i}^{[j]} \mu_{i}^{[j]}\right)$. According to Theorem 7 and Definition 5, $\boldsymbol{\Lambda}+\boldsymbol{D}_{k_{i}, k_{i}}^{\prime}$ is sign stable.

Theorem 12. Under the deterministic switching strategy (H4) of Theorem 4, while $\mu_{i}^{[j]}$ allows (H3) to hold, if $\forall i \in \overline{\mathrm{N}}, \forall j \in \overline{\mathrm{M}}$ and $\operatorname{sgn} A_{i}^{[j]}$ satisfies Theorem 11, the system in (21) is EMS sign stable.

Proof. The proof is similar to that of Theorem 10 .

Theorem 13. For the system in (21), 1-moment exponential sign stability and EMS sign stability are sign equivalent.

Proof. From Theorem 9 and Theorem 11, if $\operatorname{sgn} \bar{A}$ and $\operatorname{sgn} \tilde{A}$ are sign stable, then $\operatorname{diag}\left[\boldsymbol{A}_{i}^{[j]}+\lambda_{i}^{[j]} \mu_{i}^{[j]} \boldsymbol{I}_{n}\right]<0$ and $\operatorname{diag}\left[\boldsymbol{A}_{i}^{[j]}+\frac{1}{2} \lambda_{i}^{[j]} \mu_{i}^{[j]} \boldsymbol{I}_{n}\right]<0$. Since $\lambda_{i}^{[j]} \mu_{i}^{[j]}>0, \operatorname{diag} \boldsymbol{A}_{i}^{[j]}<0$. All $G_{A_{i}^{[j]}}$ are acyclic because $G_{R}$ is acyclic and all $A_{i}^{[j]}$ are Metzler matrices. Thus, for a DSLCTSs (21), 1-moment exponential sign stability and EMS sign stability are sign equivalent.

\section{Sign Example and Numerical Example}

For 1-moment exponential sign stability and EMS sign stability, let $\mathrm{M}=2, \mathrm{~N}=3$.

In order to satisfy condition (ii) of Theorem 9 and Theorem 11, select the original sign pattern as

$$
\boldsymbol{R}=\left[\begin{array}{ccc}
- & * & 0 \\
0 & - & 0 \\
* & * & -
\end{array}\right],
$$




$$
S=\left\{S_{1}, S_{2}, S_{3}, \ldots\right\}=\left\{\left[\begin{array}{ccc}
- & + & 0 \\
0 & - & 0 \\
+ & + & -
\end{array}\right],\left[\begin{array}{ccc}
- & + & 0 \\
0 & - & 0 \\
0 & + & -
\end{array}\right],\left[\begin{array}{ccc}
- & 0 & 0 \\
0 & - & 0 \\
+ & + & -
\end{array}\right], \ldots\right\} .
$$

Then, choose the sign matrices arbitrarily in $S$ as the sign matrices of the subsystem.

For example,

$$
\operatorname{sgn} A_{i}^{[j]}=\left\{\left[\begin{array}{ccc}
- & + & 0 \\
0 & - & 0 \\
+ & + & -
\end{array}\right],\left[\begin{array}{ccc}
- & + & 0 \\
0 & - & 0 \\
0 & + & -
\end{array}\right],\left[\begin{array}{ccc}
- & 0 & 0 \\
0 & - & 0 \\
+ & + & -
\end{array}\right]\right\}(i=1,2,3 ; j=1,2) .
$$

Assume the corresponding generators of the Markov switching signals $\sigma(t, 1)$ and $\sigma(t, 2)$ as

$$
Q^{[1]}=\left[\begin{array}{ccc}
-3 & 1 & 2 \\
2 & -4 & 2 \\
1 & 5 & -6
\end{array}\right], Q^{[2]}=\left[\begin{array}{ccc}
-4 & 3 & 1 \\
1 & -2 & 1 \\
3 & 2 & -5
\end{array}\right]
$$

The invariant distributions are $\boldsymbol{\Pi}^{[1]}=[7 / 20,2 / 5,1 / 4], \boldsymbol{\Pi}^{[2]}=[4 / 15,17 / 30,1 / 6]$. The

\begin{tabular}{|c|c|c|c|c|c|}
\hline \multicolumn{3}{|c|}{ 1-Moment Exponential Sign Stability } & \multicolumn{3}{|c|}{ EMS Sign Stability } \\
\hline$\mu_{1}^{[1]}=1.2$ & $\lambda_{1}^{[1]}=0.5$ & & $\mu_{1}^{[1]}=1.35$ & $\lambda_{1}^{[1]}=1.2$ & \multirow[b]{2}{*}{$\eta^{[1]}=-0.1$} \\
\hline$\mu_{2}^{[1]}=1.1$ & $\lambda_{2}^{[1]}=0.8$ & $\xi^{[1]}=2.4$ & $\mu_{2}^{[1]}=1.42$ & $\lambda_{2}^{[1]}=1.5$ & \\
\hline$\mu_{3}^{[1]}=1.15$ & $\lambda_{3}^{[1]}=0.7$ & & $\mu_{3}^{[1]}=1.38$ & $\lambda_{3}^{[1]}=1.8$ & \multirow{4}{*}{$\eta^{[2]}=-0.344$} \\
\hline$\mu_{1}^{[2]}=1.23$ & $\lambda_{1}^{[2]}=0.6$ & & $\mu_{1}^{[2]}=1.25$ & $\lambda_{1}^{[2]}=0.94$ & \\
\hline$\mu_{2}^{[2]}=1.18$ & $\lambda_{2}^{[2]}=0.7$ & $\xi^{[2]}=2$ & $\mu_{2}^{[2]}=1.35$ & $\lambda_{2}^{[2]}=1.1$ & \\
\hline$\mu_{3}^{[2]}=1.16$ & $\lambda_{3}^{[2]}=0.4$ & & $\mu_{3}^{[2]}=1.2$ & $\lambda_{3}^{[2]}=1.2$ & \\
\hline
\end{tabular}
initial condition is chosen as $x_{0}=[0.5,0.6,0.6]$. The other parameters are shown in Table 2 .

Table 2. The corresponding parameters of sign stability.

For 1-moment exponential sign stability, $\operatorname{diag}\left[\boldsymbol{A}_{i}^{[j]}+\lambda_{i}^{[j]} \mu_{i}^{[j]} \boldsymbol{I}\right]<0$, the ADT of 1-moment exponential sign stability is $\tau^{*}>9.95$; for EMS sign stability, $\operatorname{diag}\left[\boldsymbol{A}_{i}^{[j]}+\frac{1}{2} \lambda_{i}^{[j]} \mu_{i}^{[j]} \boldsymbol{I}\right]<0$. The numerical verification of the 1-moment exponential stability and EMS stability follows.

$$
\left(A_{1}^{[1]}\right)_{i i}<-0.4,\left(A_{2}^{[1]}\right)_{i i}<-0.9,\left(A_{3}^{[1]}\right)_{i i}<-1,\left(A_{1}^{[2]}\right)_{i i}<-1.12,\left(A_{2}^{[2]}\right)_{i i}<-0.828,
$$
$\left(A_{3}^{[2]}\right)_{i i}<-1.536$ Is used to assess the 1-moment exponential stability.

$$
\left(A_{1}^{[1]}\right)_{i i}<-0.81,\left(A_{2}^{[1]}\right)_{i i}<-1.065,\left(A_{3}^{[1]}\right)_{i i}<-1.242,\left(A_{1}^{[2]}\right)_{i i}<-0.5875,
$$
$\left(A_{2}^{[2]}\right)_{i i}<-0.7425,\left(A_{3}^{[2]}\right)_{i i}<-0.72$, is used to assess the EMS stability.

Example 1. 1-moment exponential stability.

Substituting the numerical values to verify the 1-moment exponential stability.

$$
\begin{aligned}
& A_{1}^{[1]}=\left[\begin{array}{ccc}
-1 & 3 & 0 \\
0 & -2 & 0 \\
2 & 2 & -1
\end{array}\right], A_{2}^{[1]}=\left[\begin{array}{ccc}
-2 & 3 & 0 \\
0 & -1 & 0 \\
0 & 2 & -3
\end{array}\right], A_{3}^{[1]}=\left[\begin{array}{ccc}
-3 & 0 & 0 \\
0 & -2 & 0 \\
3 & 1 & -2
\end{array}\right], \\
& A_{1}^{[2]}=\left[\begin{array}{ccc}
-2 & 1 & 0 \\
0 & -2 & 0 \\
2 & 2 & -3
\end{array}\right], A_{2}^{[2]}=\left[\begin{array}{ccc}
-1 & 2 & 0 \\
0 & -2 & 0 \\
0 & 4 & -2
\end{array}\right], A_{3}^{[2]}=\left[\begin{array}{ccc}
-2 & 0 & 0 \\
0 & -2 & 0 \\
1 & 2 & -2
\end{array}\right] .
\end{aligned}
$$


Let $\tau^{*}=10$, solving $(H 1),(H 2)$, and $(H 3)$ of Theorem 2 gives

$$
\begin{aligned}
& c_{1}^{[1]}=\left[\begin{array}{lll}
38.4437 & 580.1959 & 9.2819
\end{array}\right], c_{2}^{[1]}=\left[\begin{array}{lll}
32.6293 & 615.6027 & 9.9671
\end{array}\right], \\
& c_{3}^{[1]}=\left[\begin{array}{llll}
35.7006 & 581.3552 & 10.2330
\end{array}\right], c_{1}^{[2]}=\left[\begin{array}{lll}
52.6075 & 343.2418 & 15.6846
\end{array}\right], \\
& c_{2}^{[2]}=\left[\begin{array}{llll}
52.0306 & 335.3165 & 15.4832
\end{array}\right], c_{3}^{[2]}=\left[\begin{array}{lll}
51.1491 & 334.9320 & 15.2949
\end{array}\right] .
\end{aligned}
$$

Figures 5 and 6 present the ADT $\tau^{*}=10$ and the state trajectories, respectively.

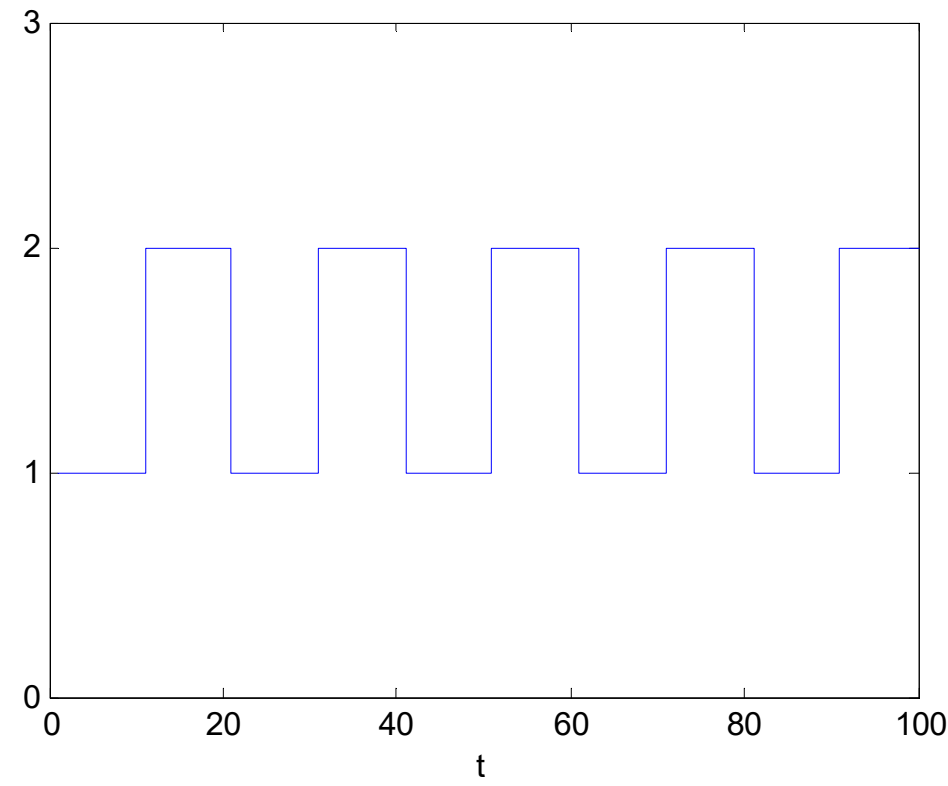

Figure 5. Switching signal $\gamma(t)$.

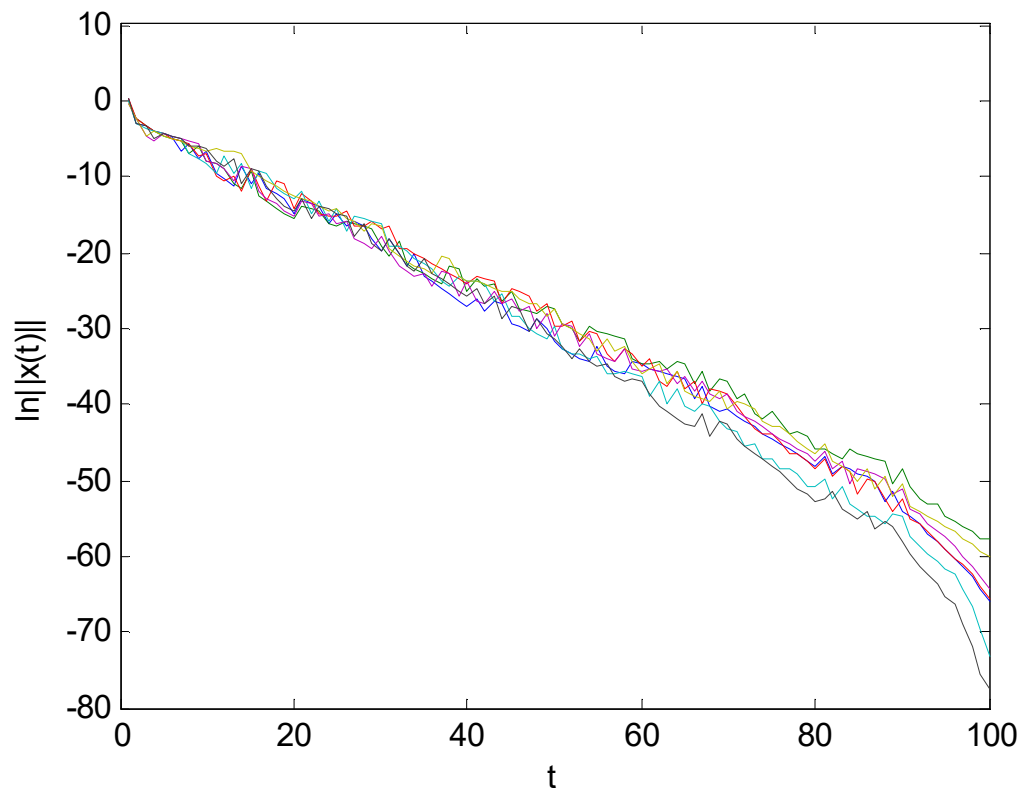

Figure 6. Seven realizations of $\ln \|x(t)\|$.

Example 2. 1-moment exponential unstability. 
Substituting the numerical values to make the first subsystem ineligible.

$$
\begin{aligned}
& A_{1}^{[1]}=\left[\begin{array}{ccc}
-0.3 & 3 & 0 \\
0 & -0.2 & 0 \\
2 & 2 & -0.1
\end{array}\right], A_{2}^{[1]}=\left[\begin{array}{ccc}
-0.2 & 3 & 0 \\
0 & -0.5 & 0 \\
0 & 2 & -0.6
\end{array}\right], A_{3}^{[1]}=\left[\begin{array}{ccc}
-0.8 & 0 & 0 \\
0 & -0.5 & 0 \\
3 & 1 & -0.9
\end{array}\right], \\
& A_{1}^{[2]}=\left[\begin{array}{ccc}
-2 & 1 & 0 \\
0 & -2 & 0 \\
2 & 2 & -3
\end{array}\right], A_{2}^{[2]}=\left[\begin{array}{ccc}
-1 & 2 & 0 \\
0 & -2 & 0 \\
0 & 4 & -2
\end{array}\right], A_{3}^{[2]}=\left[\begin{array}{ccc}
-2 & 0 & 0 \\
0 & -2 & 0 \\
1 & 2 & -2
\end{array}\right] .
\end{aligned}
$$

Let $\tau^{*}=10$, Figures 5 and 7 present the ADT $\tau^{*}=10$ and the state trajectories, respectively.

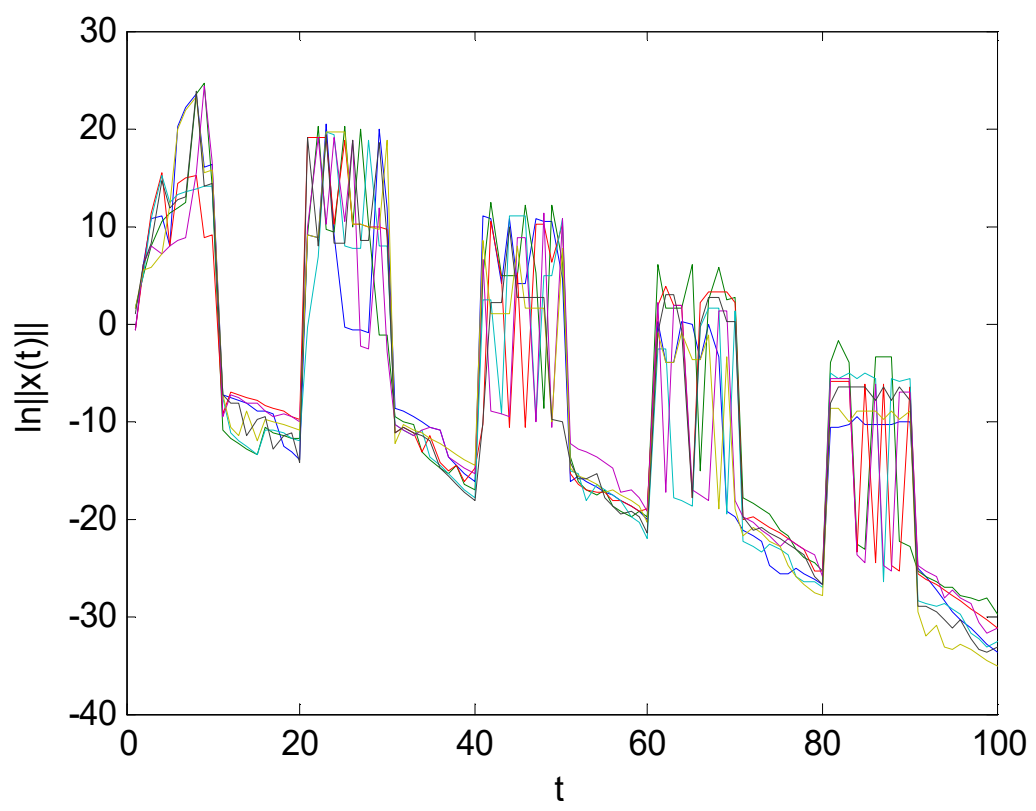

Figure 7. Seven realizations of $\ln \|x(t)\|$.

Because the first subsystem matrices do not meet the conditions, when switching to the first subsystem it can be seen from Figure 7 that the state trajectories are unstable.

Example 3. EMS stability.

Substituting the numerical values to evaluate the EMS stability.

$$
\begin{aligned}
& A_{1}^{[1]}=\left[\begin{array}{ccc}
-1 & 3 & 0 \\
0 & -2 & 0 \\
1 & 2 & -1
\end{array}\right], A_{2}^{[1]}=\left[\begin{array}{ccc}
-1 & 3 & 0 \\
0 & -3 & 0 \\
0 & 2 & -3
\end{array}\right], A_{3}^{[1]}=\left[\begin{array}{ccc}
-3 & 0 & 0 \\
0 & -2 & 0 \\
1 & 2 & -2
\end{array}\right], \\
& A_{1}^{[2]}=\left[\begin{array}{ccc}
-1 & 2 & 0 \\
0 & -2 & 0 \\
2 & 2 & -1
\end{array}\right], A_{2}^{[2]}=\left[\begin{array}{ccc}
-2 & 2 & 0 \\
0 & -3 & 0 \\
0 & 3 & -2
\end{array}\right], A_{3}^{[2]}=\left[\begin{array}{ccc}
-2 & 0 & 0 \\
0 & -2 & 0 \\
1 & 2 & -2
\end{array}\right] .
\end{aligned}
$$

Solving (H1) and (H2) of Theorem 4 yields

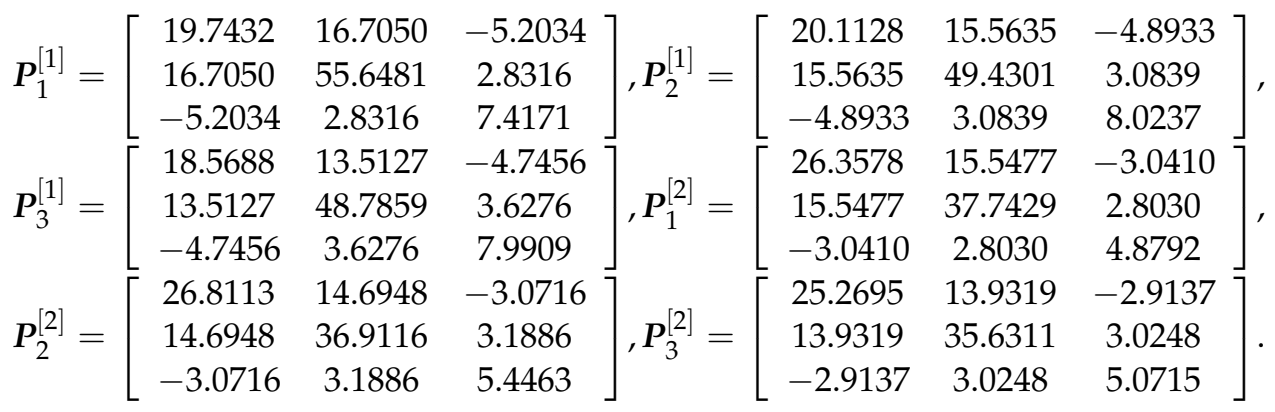


Figures 8 and 9 give the deterministic switching signal $\gamma(t)$ and the state responses, respectively.

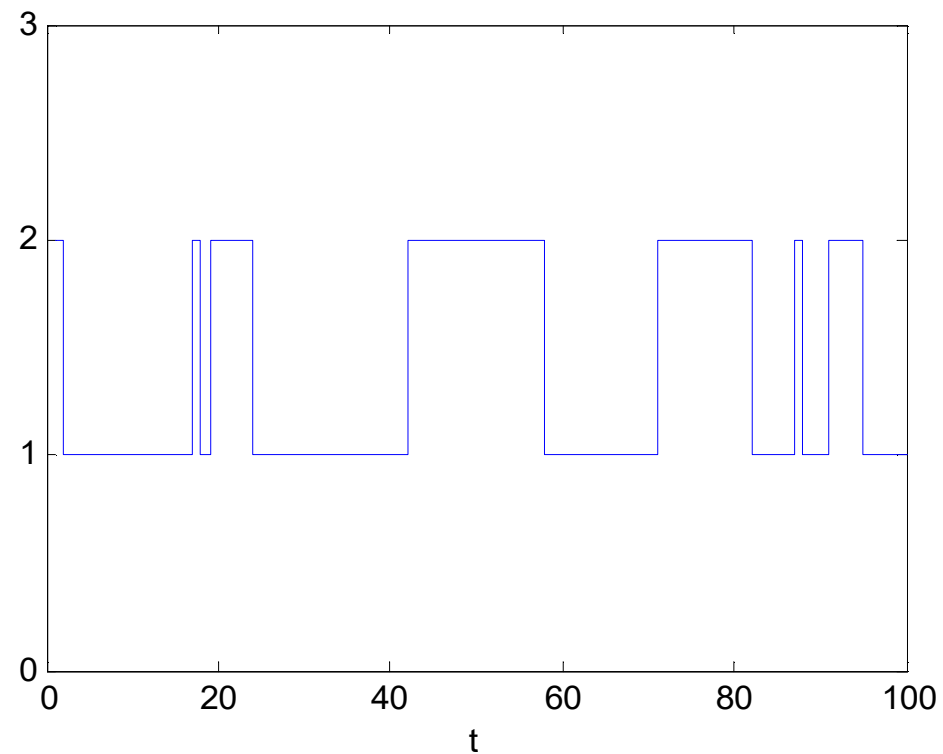

Figure 8. Switching signal $\gamma(t)$.

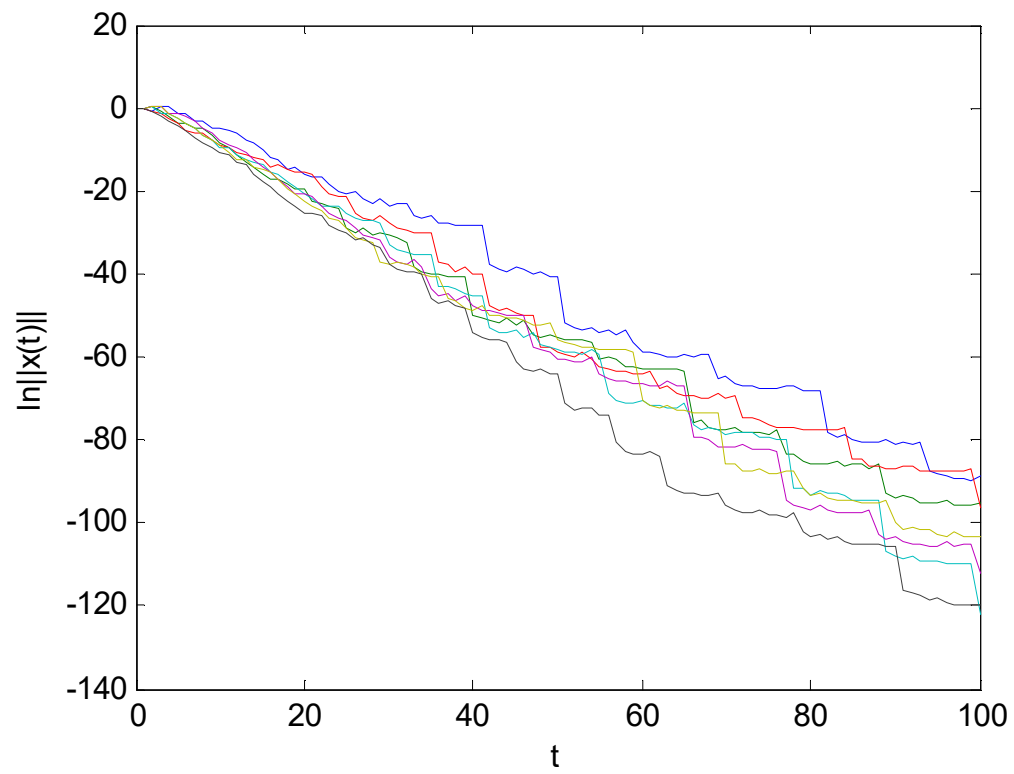

Figure 9. Seven realizations of $\ln \|x(t)\|$.

Example 4. EMS stability without the deterministic switching.

Substituting the numerical values to make the second subsystem ineligible

$$
\begin{aligned}
& A_{1}^{[1]}=\left[\begin{array}{ccc}
-1 & 3 & 0 \\
0 & -2 & 0 \\
1 & 2 & -1
\end{array}\right], A_{2}^{[1]}=\left[\begin{array}{ccc}
-1 & 3 & 0 \\
0 & -3 & 0 \\
0 & 2 & -3
\end{array}\right], A_{3}^{[1]}=\left[\begin{array}{ccc}
-3 & 0 & 0 \\
0 & -2 & 0 \\
1 & 2 & -2
\end{array}\right], \\
& A_{1}^{[2]}=\left[\begin{array}{ccc}
-0.1 & 2 & 0 \\
0 & -0.2 & 0 \\
2 & 2 & -0.1
\end{array}\right], A_{2}^{[2]}=\left[\begin{array}{ccc}
-0.2 & 2 & 0 \\
0 & -0.3 & 0 \\
0 & 3 & -0.2
\end{array}\right], A_{3}^{[2]}=\left[\begin{array}{ccc}
-0.2 & 0 & 0 \\
0 & -0.2 & 0 \\
1 & 2 & -0.2
\end{array}\right] .
\end{aligned}
$$


Figures 10 and 11 give the deterministic switching signal $\gamma(t)$ and the state responses, respectively.

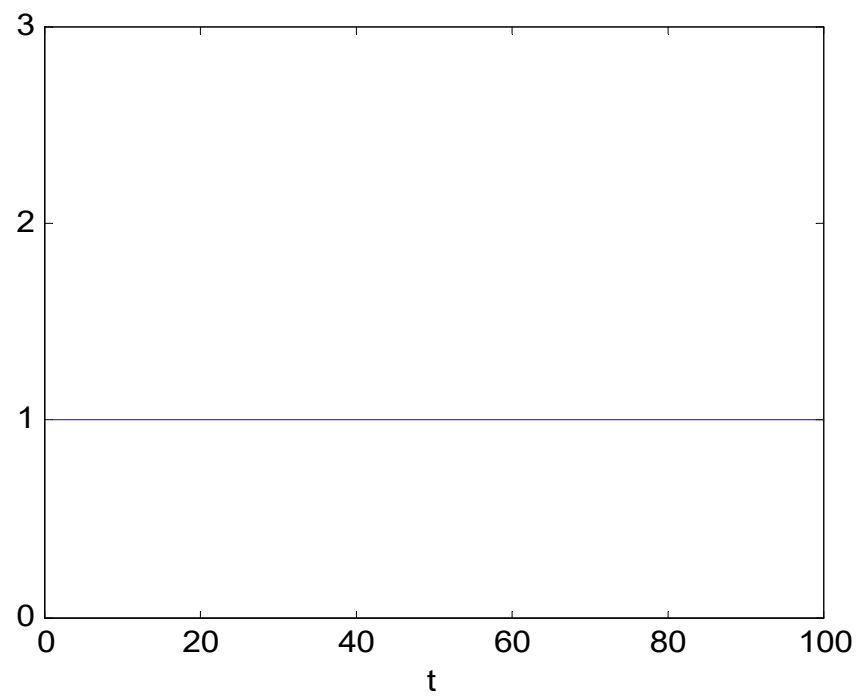

Figure 10. Switching signal $\gamma(t)$.

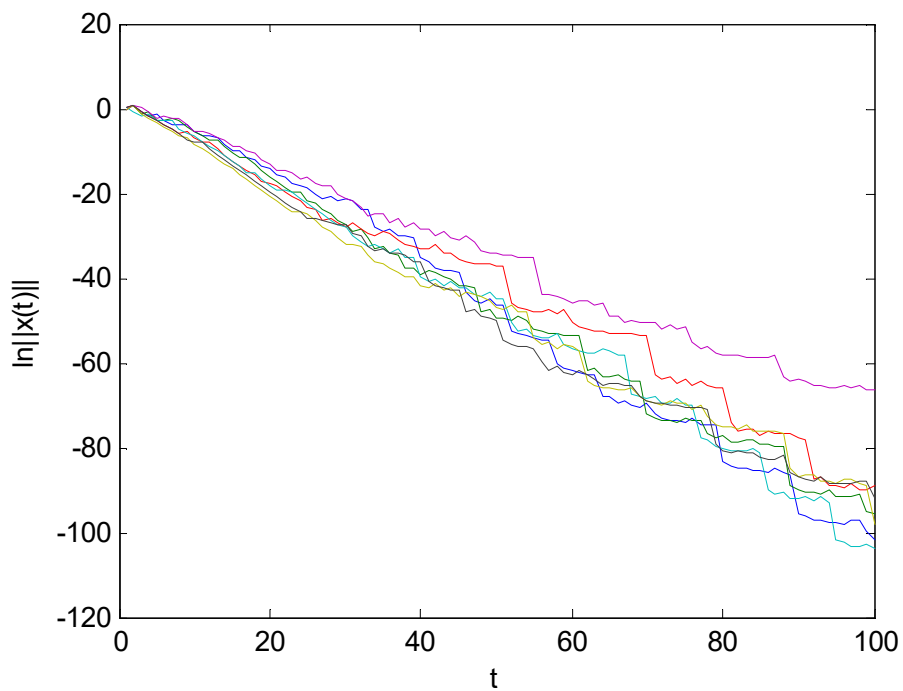

Figure 11. Seven realizations of $\ln \|x(t)\|$.

Because the second subsystem matrices do not meet the conditions, according to preset deterministic switching strategy, it can be seen from Figure 10 that the system only runs on the first stable subsystem.

\section{Conclusions}

The ADT and the preset deterministic switching strategy are employed to cope with the 1-moment exponential stability and EMS stability for DSLCTSs. The derivation of the sufficient stability condition in the form of LMI are reached in this research. 1-moment exponential stability cannot inevitably imply EMS stability. If 1-moment exponential stability is induced by a DSLCTS for all possible realizations of sign matrices, it also has EMS stability. This means that both concepts are not equivalent for a specific realization of a DSLCTS; they are structurally equivalent. Thus, structural concepts of stability can be introduced utilizing the sign stability concept to equate the traditional concepts of 1-moment exponential stability and EMS stability. The structural notions of stability for 
dual switching linear discrete-time systems could be further investigated as a future research subject.

Author Contributions: Methodology, L.L. and F.L.; analysis, F.L.; writing-original draft preparation, L.L.; writing-review and editing, F.L., L.M. and Q.M. All authors have read and agreed to the published version of the manuscript.

Funding: This work was supported by National Natural Science Foundation under Grants (61813006, 61973329), Key Projects of Basic Research Program of Guizhou Province under Grant (20191416), Guizhou Education Department Youth Science and Technology Talent Growth Project (QianJiaoHeKY-Zi[2021]239).

Institutional Review Board Statement: Not applicable.

Informed Consent Statement: Not applicable.

Data Availability Statement: The work is not based on any data.

Conflicts of Interest: The authors declare no conflict of interest.

\section{Appendix A}

Proof. Define $V_{i}^{[j]}(\boldsymbol{x})=\left(\boldsymbol{c}_{i}^{[j]}\right)^{T} x$ for each $i \in \overline{\mathrm{N}}$ and $j \in \overline{\mathrm{M}}$. Suppose that $\gamma(t)=\gamma_{k} \in \overline{\mathrm{M}}$ for $t \in\left[t_{k}, t_{k+1}\right)$ and $\gamma\left(t_{k+1}\right) \neq \gamma_{k}$. In $\left[t_{k}, t_{k+1}\right)$, none of the deterministic switching occurring is assumed. The switching sequence (SS) of the switching signal by Markov process $\sigma\left(t, \gamma_{k}\right)$ in the interval $\left[t_{k}, t_{k+1}\right)$ denoted by $t_{k}=\tau_{0}<\tau_{1}<\ldots<\tau_{l}<t_{k+1}$ is assumed. Namely, $t \in\left[\tau_{v}, \tau_{v+1}\right) \subset\left[t_{k}, t_{k+1}\right)$ for, $v \in\{0,1,2, \ldots, l\}, l<\infty$, and $\tau_{l+1}=t_{k+1}$. Now, based on (H1) and (H2), the following inequality is obtained for $\forall t \in\left[\tau_{l}, t_{k+1}\right)$

$$
\left(\underset{\sigma_{\sigma_{r}}^{\gamma_{g}}}{\left[\gamma_{g}\right]}\right)^{T} x(t) \leq \xi^{\left[\gamma_{k}\right]}\left(\boldsymbol{c}_{\sigma_{l}^{\gamma_{k}}}^{\left[\gamma_{k}\right]}\right)^{T} x(t)<\xi^{\left[\gamma_{k}\right]}\left(\boldsymbol{c}_{\sigma_{l}^{\gamma_{k}}}^{\left[\gamma_{k}\right]}\right)^{T} x\left(\tau_{l}\right) \times \exp \left\{-\lambda_{\sigma_{l}^{\gamma_{k}}}^{\left[\gamma_{k}\right]} \times\left(t-\tau_{l}\right)\right\}
$$

Assuming that $\left[t_{k}, t_{k+1}\right)=\left[t_{k}, \tau_{1}\right) \cup\left[\tau_{1}, \tau_{2}\right) \cup \ldots \cup\left[\tau_{1}, t_{k+1}\right),(H 3)$ is employed this leads to $\left(c_{\sigma_{r}^{\gamma g}}^{\left[\gamma_{g}\right]}\right)^{T} x(t)<\xi^{\left[\gamma_{k}\right]}\left(c_{\sigma_{\sigma_{k}}^{\gamma_{k}}}^{\left[\gamma_{k}\right]}\right)^{T} x\left(\tau_{l}\right) \times \exp \left\{-\lambda_{\sigma_{l}^{\gamma / k}}^{\left[\gamma_{k}\right]} \times\left(t-\tau_{l}\right)\right\}$ $\leq \xi^{\left[\gamma_{k}\right]} \cdot \mu_{\sigma_{l}^{\gamma_{k}}}^{\left[\gamma_{k}\right]} \times\left(c_{\sigma_{l-1}^{\gamma_{k}}}^{\left[\gamma_{k}\right]}\right)^{T} x\left(\tau_{l}\right) \times \exp \left\{-\lambda_{\sigma_{l}^{\gamma_{k}}}^{\left[\gamma_{k}\right]} \times\left(t-\tau_{l}\right)\right\}$.

$<\xi^{\left[\gamma_{k}\right]} \cdot \mu_{\sigma_{l}^{\gamma_{k}}}^{\left[\gamma_{k}\right]} \times\left[\left(c_{\sigma_{l-1}^{\gamma_{k}}}^{\left[\gamma_{k}\right]}\right)^{T} x\left(\tau_{l-1}\right) \times \exp \left\{-\lambda_{\sigma_{l-1}^{\gamma_{k}}}^{\left[\gamma_{k}\right]} \times\left(\tau_{l}-\tau_{l-1}\right)\right\}\right] \times \exp \left\{-\lambda_{\sigma_{l}^{\gamma_{k}}}^{\left[\gamma_{k}\right]} \times\left(t-\tau_{l}\right)\right\}$

$=\xi^{\left[\gamma_{k}\right]} \cdot \mu_{\sigma_{l}^{\gamma_{k}}}^{\left[\gamma_{k}\right]} \times\left(\boldsymbol{c}_{\sigma_{l-1}^{\gamma_{k}}}^{\left[\gamma_{k}\right]}\right)^{T} x\left(\tau_{l-1}\right) \times \exp \left\{-\lambda_{\sigma_{l-1}^{\gamma_{k}}}^{\left[\gamma_{k}\right]} \times\left(\tau_{l}-\tau_{l-1}\right)-\lambda_{\sigma_{l}^{\gamma_{k}}}^{\left[\gamma_{k}\right]} \times\left(t-\tau_{l}\right)\right\}$

$<\xi^{\left[\gamma_{k}\right]} \cdot \prod_{v=1}^{l} \mu_{\sigma_{v}^{\gamma_{k}}}^{\left[\gamma_{k}\right]} \times\left(\boldsymbol{c}_{\sigma_{t_{k}}^{\gamma_{k}}}^{\left[\gamma_{k}\right]}\right)^{T} x\left(t_{k}\right) \times \exp \left\{-\lambda_{\sigma_{l}^{\gamma_{k}}}^{\left[\gamma_{k}\right]} \times\left(t-\tau_{l}\right)-\sum_{v=1}^{l-1}\left[\lambda_{\sigma_{v}^{\gamma_{k}}}^{\left[\gamma_{k}\right]} \times\left(\tau_{v+1}-\tau_{v}\right)\right]\right\}$

Therefore, for any $t \in\left[t_{k}, t_{k+1}\right)$

$$
\begin{aligned}
& \left(\underset{\sigma_{r}^{\gamma g}}{\left[\gamma_{g}\right]}\right)^{T} x(t)<\xi^{\left[\gamma_{k}\right]} \cdot \prod_{\sigma_{v}^{\gamma_{k}}=1}^{N}\left(\mu_{\sigma_{v}^{\gamma} \gamma_{k}}^{\left[\gamma_{k}\right]}\right)^{N_{\sigma_{v}^{\gamma k}}^{\left[\gamma_{k}\right]}\left(t_{k}, t\right)} \times\left(\boldsymbol{c}_{\sigma_{t_{k}}^{\gamma_{k}}}^{\left[\gamma_{k}\right]}\right)^{T} x\left(t_{k}\right) \times \exp \left\{-\int_{t_{k}}^{t} \lambda_{\sigma\left(s, \gamma_{k}\right)}^{\left[\gamma_{k}\right]} d s\right\} . \\
& =\xi^{\left[\gamma_{k}\right]} \cdot\left(c_{\sigma_{t_{k}}^{\gamma_{k}}}^{\left[\gamma_{k}\right]}\right)^{T} x\left(t_{k}\right) \times \exp \left\{\sum_{\sigma_{v}^{\gamma_{k}}=1}^{N} N_{\sigma_{v}^{\gamma_{k}}}^{\left[\gamma_{k}\right]}\left(t_{k}, t\right) \times \ln \left(\mu_{\sigma_{v}^{\gamma}}^{\left[\gamma_{k}\right]}\right)\right\} \times \\
& \exp \left\{-\sum_{\sigma_{v}^{\gamma_{k}}=1}^{N} \lambda_{\sigma_{v}^{\gamma_{k}}}^{\left[\gamma_{k}\right]} \times T_{\sigma_{v}^{\gamma_{k}}}^{\left[\gamma_{k}\right]}\left(t_{k}, t\right)\right\}
\end{aligned}
$$


Both (7) and (8) conclude that for $\forall i \in \overline{\mathrm{N}}, \forall j \in \overline{\mathrm{M}}$ and $\varepsilon$ (a positive constant)

$$
T_{\sigma_{v}^{\gamma} \gamma_{k}}^{\left[\gamma_{k}\right]}\left(t_{k}, t\right) \leq\left(\pi_{\sigma_{v}^{\gamma_{k}}}^{\left[\gamma_{k}\right]}+\varepsilon\right)\left(t-t_{k}\right)
$$

and

$$
N_{\sigma_{v}^{\gamma_{k}}}^{\left[\gamma_{k}\right]}\left(t_{k}, t\right) \leq\left(\pi_{\sigma_{v}^{\gamma} \gamma_{k}}^{\left[\gamma_{k}\right]}\left|q_{\sigma_{v}^{\gamma_{k}, \sigma_{v}}}^{\left[\gamma_{k}\right]}\right|+\varepsilon\right)\left(t-t_{k}\right)
$$

where $t_{0}=0, t_{k+1}=t$.

Utilizing (A3)-(A5), the following inequality could be reached for $\forall t>0$

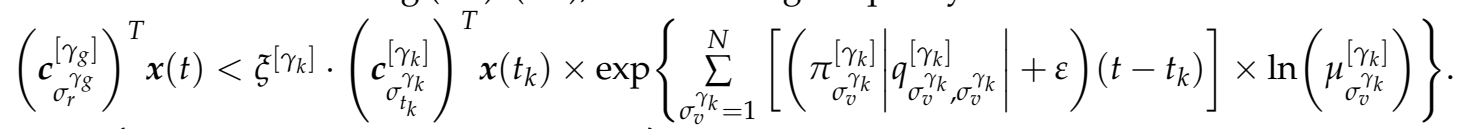

$$
\begin{aligned}
& \times \exp \left\{-\sum_{\sigma_{v}^{\gamma_{k}}=1}^{N} \lambda_{\sigma_{v}^{\gamma} \gamma_{k}}^{\left[\gamma_{k}\right]} \times\left(\pi_{\sigma_{v}^{\gamma_{k}}}^{\left[\gamma_{k}\right]}+\varepsilon\right)\left(t-t_{k}\right)\right\}
\end{aligned}
$$

Taking logarithms, on both sides of the above inequality, it follows that

$$
\begin{aligned}
& \ln \left[\left(\boldsymbol{c}_{\sigma_{r}}^{\left[\gamma_{g}\right]}\right)^{T} x(t)\right]<\ln \xi^{\left[\gamma_{k}\right]}+\ln \left[\left(\boldsymbol{c}_{\sigma_{t_{k}}^{\gamma_{k}}}^{\left[\gamma_{k}\right]}\right)^{T} x\left(t_{k}\right)\right] \\
& +\sum_{\sigma_{v}^{\gamma_{k}}=1}^{N}\left[\left(\pi_{\sigma_{v}^{\gamma_{k}}}^{\left[\gamma_{k}\right]}\left|q_{\sigma_{v}^{\gamma_{k}}, \sigma_{v}^{\gamma_{k}}}^{\left[\gamma_{k}\right]}\right|+\varepsilon\right)\left(t-t_{k}\right)\right] \times \ln \left(\mu_{\sigma_{v}^{\gamma_{k}}}^{\left[\gamma_{k}\right]}\right)-\sum_{\sigma_{v}^{\gamma_{k}}=1}^{N} \lambda_{\sigma_{v}^{\gamma_{k}}}^{\left[\gamma_{k}\right]} \times\left(\pi_{\sigma_{v}^{\gamma_{k}}}^{\left[\gamma_{k}\right]}+\varepsilon\right)\left(t-t_{k}\right) \\
& \operatorname{Let} \theta^{\left[\gamma_{k}\right]}(\varepsilon)=\sum_{\sigma_{v}^{\gamma_{k}}=1}^{\mathrm{N}}\left(\pi_{\sigma_{v}^{\gamma_{k}}}^{\left[\gamma_{k}\right]}\left|q_{\sigma_{v}^{\gamma_{k}}, \sigma_{v}^{\gamma_{k}}}^{\left[\gamma_{k}\right]}\right|+\varepsilon\right) \times \ln \left(\mu_{\sigma_{v}^{\gamma_{k}}}^{\left[\gamma_{k}\right]}\right), \rho^{\left[\gamma_{k}\right]}(\varepsilon)=-\sum_{\sigma_{v}^{\gamma_{k}=1}}^{\mathrm{N}} \lambda_{\sigma_{v}^{\gamma_{k}}}^{\left[\gamma_{\gamma^{\prime}}\right]} \times\left(\pi_{\sigma_{v}^{\gamma_{k}}}^{\left[\gamma_{k}\right]}+\varepsilon\right) \text {, then } \\
& \ln \left[\left(\boldsymbol{c}_{\sigma_{r}^{\gamma g}}^{\left[\gamma_{g}\right]}\right)^{T} x(t)\right]<\ln \xi^{\left[\gamma_{k}\right]}+\ln \left[\left(\boldsymbol{c}_{\sigma_{t_{k}}^{\gamma_{k}}}^{\left[\gamma_{k}\right]}\right)^{T} x\left(t_{k}\right)\right]+\left(\theta^{\left[\gamma_{k}\right]}(\varepsilon)+\rho^{\left[\gamma_{k}\right]}(\varepsilon)\right)\left(t-t_{k}\right)
\end{aligned}
$$

In $\left[t_{0}, t\right)$, the total visit time of the subsystem and activation number is defined by $T^{[j]}\left(t_{0}, t\right)$ and $N^{[j]}\left(t_{0}, t\right)$, respectively.

Similar to the above process, we derive

$$
\begin{aligned}
& \ln \left[\left(\boldsymbol{c}_{\sigma_{r}^{\gamma g}}^{\left[\gamma_{g}\right]}\right)^{T} \boldsymbol{x}(t)\right]<\sum_{\gamma_{k}=1}^{\mathrm{M}} N^{\left[\gamma_{k}\right]}\left(t_{0}, t\right) \cdot \ln \xi^{\left[\gamma_{k}\right]}+ \\
& \sum_{\gamma_{k}=1}^{\mathrm{M}}\left[\left(\theta^{\left[\gamma_{k}\right]}(\varepsilon)+\rho^{\left[\gamma_{k}\right]}(\varepsilon)\right) \cdot T^{\left[\gamma_{k}\right]}\left(t_{0}, t\right)\right]+\ln \left[\left(\boldsymbol{c}_{\sigma_{t_{0}}^{\gamma \gamma_{0}}}^{\left[\gamma_{0}\right]}\right)^{T} x\left(t_{0}\right)\right]
\end{aligned}
$$

where $\bar{w}(\varepsilon)=\max _{\gamma_{k} \in \overline{\mathrm{M}}}\left\{\theta^{\left[\gamma_{k}\right]}(\varepsilon)+\rho^{\left[\gamma_{k}\right]}(\varepsilon)\right\}$, and $\bar{\xi}=\max _{\gamma_{k} \in \overline{\mathrm{M}}}\left\{\ln \xi^{\left[\gamma_{k}\right]}\right\}$, Let $\theta^{\left[\gamma_{k}\right]}=\sum_{\sigma_{v}^{\gamma_{k}}=1}^{\mathrm{N}} \pi_{\sigma_{v}^{\gamma_{k}}}^{\left[\gamma_{k}\right]}\left|q_{\sigma_{v}^{\gamma_{k}}, \sigma_{v}^{\gamma_{k}}}^{\left[\gamma_{k}\right]}\right| \times \ln \left(\mu_{\sigma_{v}^{\gamma_{k}}}^{\left[\gamma_{k}\right]}\right), \quad \rho^{\left[\gamma_{k}\right]}(\varepsilon)=-\sum_{\sigma_{v}^{\gamma_{k}}=1}^{\mathrm{N}} \lambda_{\sigma_{v}^{\gamma_{k}}}^{\left[\gamma_{k}\right]} \times \pi_{\sigma_{v}^{\gamma_{k}}}^{\left[\gamma_{k}\right]}$, $\bar{w}=\max _{\gamma_{k} \in \bar{M}}\left\{\theta^{\left[\gamma_{k}\right]}+\rho^{\left[\gamma_{k}\right]}\right\}$, since $\ln \mu_{i}^{[j]}<\lambda_{i}^{[j]}$, then $\bar{w}(\varepsilon)<\bar{w}$,

$\mathrm{E}\left(\ln \left[\left(\boldsymbol{c}_{\sigma_{r}^{\gamma_{\mathcal{g}}}}^{\left[\gamma_{g}\right]}\right)^{T} x(t)\right]\right)<\sum_{\gamma_{k}=1}^{M} N^{\left[\gamma_{k}\right]}\left(t_{0}, t\right) \cdot \bar{\xi}+\bar{w}(\varepsilon) \cdot \sum_{\gamma_{k}=1}^{M} T^{\left[\gamma_{k}\right]}\left(t_{0}, t\right)+\mathrm{E}\left(\ln \left[\left(c_{\sigma_{t_{0}}^{\gamma_{0}}}^{\left[\gamma_{0}\right]}\right)^{T} x\left(t_{0}\right)\right]\right)$

$<\sum_{\gamma_{k}=1}^{M} N^{\left[\gamma_{k}\right]}\left(t_{0}, t\right) \cdot \bar{\xi}+\bar{w} \cdot \sum_{\gamma_{k}=1}^{M} T^{\left[\gamma_{k}\right]}\left(t_{0}, t\right)+\mathrm{E}\left(\ln \left[\left(\boldsymbol{c}_{\sigma_{t_{0}}^{\gamma_{0}}}^{\left[\gamma_{0}\right]}\right)^{T} x\left(t_{0}\right)\right]\right)$

The following inequalities can be obtained from Definition 2.

$$
\phi\left(t_{0}, t\right) \leq N_{0}+\frac{t-t_{0}}{\tau^{*}}
$$




$$
\begin{aligned}
& \text { where } \phi\left(t_{0}, t\right)=\sum_{\gamma_{k}=1}^{\mathrm{M}}\left[N^{\left[\gamma_{k}\right]}\left(t_{0}, t\right)\right], \sum_{\gamma_{k}=1}^{\mathrm{M}}\left[T^{\left[\gamma_{k}\right]}\left(t_{0}, t\right)\right]=t-t_{0} \text {. } \\
& \mathrm{E}\left(\ln \left[\left(\boldsymbol{c}_{\sigma_{r}^{\gamma g}}^{\left[\gamma_{g}\right]}\right)^{T} x(t)\right]\right)<N_{0} \cdot \bar{\xi}+\left(\frac{\bar{\xi}}{\tau^{*}}+\bar{w}\right) \cdot\left(t-t_{0}\right)+\mathrm{E}\left(\ln \left[\left(c_{\sigma_{t_{0}}^{\gamma}}^{\left[\gamma_{0}\right]}\right)^{T} x\left(t_{0}\right)\right]\right) \\
& \mathrm{E}\left(\left(\boldsymbol{c}_{\sigma_{r}^{\gamma g}}^{\left[\gamma_{g}\right]}\right)^{T} x(t)\right)<\exp \left\{N_{0} \cdot \bar{\xi}\right\} \cdot \exp \left\{\left(\frac{\bar{\xi}}{\tau^{*}}+\bar{w}\right) \cdot\left(t-t_{0}\right)\right\} \cdot \mathrm{E}\left[\left(c_{\sigma_{t_{0}}^{\gamma}}^{\left[\gamma_{0}\right]}\right)^{T} x\left(t_{0}\right)\right] \\
& \text { As } c_{i}^{[j]}>1, i=1,2, \ldots, \mathrm{N} \text {, } \\
& \mathrm{E}\left[1^{T} x(t)\right]<\mathrm{E}\left(\left(c_{\sigma_{r}^{\gamma g}}^{\left[\gamma_{g}\right]}\right)^{T} x(t)\right)< \\
& \frac{\exp \left\{N_{0} \cdot \bar{\xi}\right\} \cdot \mathrm{E}\left[\left(\begin{array}{c}
c_{\left.\gamma_{0}\right]}^{\left[\gamma_{0}\right]} \\
\sigma_{t_{0}}^{\gamma_{0}}
\end{array}\right)^{T} x\left(t_{0}\right)\right]}{\left\|x\left(t_{0}\right)\right\|} \times \exp \left\{\left(\frac{\bar{\xi}}{\tau^{*}}+\bar{w}\right) \cdot\left(t-t_{0}\right)\right\} \times\left\|x\left(t_{0}\right)\right\|
\end{aligned}
$$

As $x(t)$ is a nonnegative vector, then $\|x(t)\| \leq 1^{T} x(t)$,

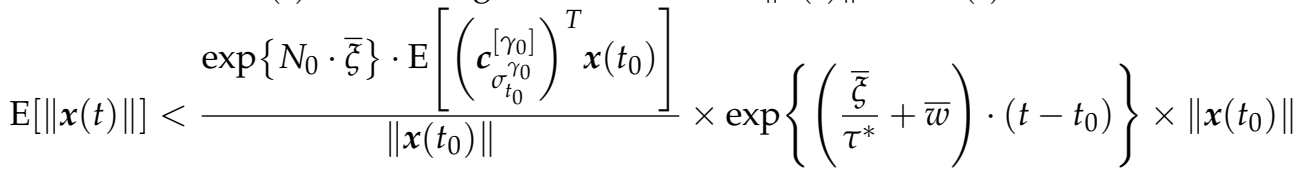

Definition 1 and (H4) imply that System (1) is 1-moment exponentially stable.

\section{Appendix B}

Proof. Define $V_{i}^{[j]}(\boldsymbol{x})=\boldsymbol{x}^{T} \boldsymbol{P}_{i}^{[j]} \boldsymbol{x}$, for each $i \in \overline{\mathrm{N}}$ and $j \in \overline{\mathrm{M}}$. Assume that $\gamma(t)=\gamma_{k} \in \overline{\mathrm{M}}$ for $t \in\left[t_{k}, t_{k+1}\right)$ and $\gamma\left(t_{k+1}\right) \neq \gamma_{k}$. None of the deterministic switching existing in the interval $\left[t_{k}, t_{k+1}\right)$ is assumed. The SS of the switching signal determined by the Markov process $\sigma\left(t, \gamma_{k}\right)$ for $\left[t_{k}, t_{k+1}\right)$, denoted by $t_{k}=\tau_{0}<\tau_{1}<\ldots<\tau_{l}<t_{k+1}$, is assumed. Namely, $\sigma\left(t, \gamma_{k}\right)=\sigma_{v}^{\gamma_{k}} \in \overline{\mathrm{N}}$ for $t \in\left[\tau_{v}, \tau_{v+1}\right) \subset\left[t_{k}, t_{k+1}\right), v \in\{0,1,2, \ldots, l\}, l<\infty$, where $\tau_{l+1}=t_{k+1}$. Utilizing $(H 1)$ and $(H 2)$, it is derived for $\forall t \in\left[\tau_{l}, t_{k+1}\right)$ and repeating the process,

$$
\begin{aligned}
& \boldsymbol{x}^{T}(t) \boldsymbol{P}_{\sigma_{l}^{\gamma_{k}}}^{\left[\gamma_{k}\right]} \boldsymbol{x}(t)<\boldsymbol{x}^{T}\left(\tau_{l}\right) \boldsymbol{P}_{\sigma_{l}^{\gamma_{k}}}^{\left[\gamma_{k}\right]} \boldsymbol{x}\left(\tau_{l}\right) \times \exp \left\{-\lambda_{\sigma_{l}^{\gamma_{k}}}^{\left[\gamma_{k}\right]} \times\left(t-\tau_{l}\right)\right\} \\
& <\mu_{\sigma_{l}^{\gamma_{k}}}^{\left[\gamma_{k}\right]} \boldsymbol{x}^{T}\left(\tau_{l}\right) \boldsymbol{P}_{\sigma_{l-1}^{\gamma_{k}}}^{\left[\gamma_{k}\right]} \boldsymbol{x}\left(\tau_{l}\right) \times \exp \left\{-\lambda_{\sigma_{l}^{\gamma_{k}}}^{\left[\gamma_{k}\right]} \times\left(t-\tau_{l}\right)\right\} \\
& <\mu_{\sigma_{l}^{\gamma_{k}}}^{\left[\gamma_{k}\right]}\left[\boldsymbol{x}^{T}\left(\tau_{l-1}\right) \boldsymbol{P}_{\sigma_{l-1}^{\gamma_{k}}}^{\left[\gamma_{k}\right]} \boldsymbol{x}\left(\tau_{l-1}\right) \times \exp \left\{-\lambda_{\sigma_{l-1}^{\gamma_{k}}}^{\left[\gamma_{k}\right]} \times\left(\tau_{l}-\tau_{l-1}\right)\right\}\right] \times \exp \left\{-\lambda_{\sigma_{l}^{\gamma_{k}}}^{\left[\gamma_{\gamma^{\prime}}\right]} \times\left(t-\tau_{l}\right)\right\} \\
& =\mu_{\sigma_{l}^{\gamma_{k}}}^{\left[\gamma_{k}\right]} \boldsymbol{x}^{T}\left(\tau_{l-1}\right) \boldsymbol{P}_{\sigma_{l-1}^{\gamma_{k}}}^{\left[\gamma_{k}\right]} \boldsymbol{x}\left(\tau_{l-1}\right) \times \exp \left\{-\lambda_{\sigma_{l-1}^{\gamma_{k}}}^{\left[\gamma_{k}\right]} \times\left(\tau_{l}-\tau_{l-1}\right)-\lambda_{\sigma_{l}^{\gamma_{k}}}^{\left[\gamma_{\gamma_{k}}\right]} \times\left(t-\tau_{l}\right)\right\} \\
& <\ldots \\
& <\boldsymbol{x}^{T}\left(t_{k}\right) \boldsymbol{P}_{\sigma_{0}^{\gamma_{k}}}^{\left[\gamma_{k}\right]} \boldsymbol{x}\left(t_{k}\right) \times \prod_{v=0}^{l} \mu_{\sigma_{v}^{\gamma_{k}}}^{\left[\gamma_{k}\right]} \times \exp \left\{-\lambda_{\sigma_{l}^{\gamma_{k}}}^{\left[\gamma_{k}\right]} \times\left(t-\tau_{l}\right)-\sum_{v=0}^{l-1}\left[\lambda_{\sigma_{v}^{\gamma_{k}}}^{\left[\gamma_{k}\right]} \times\left(\tau_{v+1}^{\gamma_{k}}-\tau_{v}\right)\right]\right\}
\end{aligned}
$$

Thus, for each $t \in\left[t_{k}, t_{k+1}\right)$,

$$
\begin{aligned}
& \boldsymbol{x}^{T}(t) \boldsymbol{P}_{\sigma\left(t, \gamma_{k}\right)}^{\left[\gamma_{k}\right]} \boldsymbol{x}(t)<\boldsymbol{x}^{T}\left(t_{k}\right) \boldsymbol{P}_{\sigma\left(t_{k}, \gamma_{k}\right)}^{\left[\gamma_{k}\right]} \boldsymbol{x}\left(t_{k}\right) \times \prod_{\sigma_{v}^{\gamma_{k}}=0}^{l}\left(\mu_{\sigma_{v}}^{\left[\gamma_{k}\right]}\right)^{N_{\sigma_{v}}^{\left[\gamma_{k}\right]}\left(t_{k}, t\right)} \times \exp \left\{-\int_{t_{k}}^{t} \lambda_{\sigma\left(s, \gamma_{k}\right)}^{\left[\gamma_{k}\right]} d s\right\} \\
& =x^{T}\left(t_{k}\right) \boldsymbol{P}_{\sigma\left(t_{k}, \gamma_{k}\right)}^{\left[\gamma_{k}\right]} x\left(t_{k}\right) \times \exp \left\{\sum_{\sigma_{v}^{\gamma_{k}}=0}^{N}\left(N_{\sigma_{v}^{\prime} k}^{\left[\gamma_{k}\right]}\left(t_{k}, t\right) \times \ln \mu_{\sigma_{v}^{\gamma_{k}}}^{\left[\gamma_{k}\right]}\right)\right\} \times \exp \left\{-\int_{t_{k}}^{t} \lambda_{\sigma\left(s, \gamma_{k}\right)}^{\left[\gamma_{k}\right]} d s\right\}
\end{aligned}
$$

Using (H3), we can select positive constant $\varepsilon$ such that for $\forall j \in \overline{\mathrm{M}}$

$$
\sum_{i=1}^{\mathrm{N}}\left[\pi_{i}^{[j]}\left(\left|q_{i i}^{[j]}\right| \ln \left(\mu_{i}^{[j]}\right)-\lambda_{i}^{[j]}\right)+\left(\ln \left(\mu_{i}^{[j]}\right)-\lambda_{i}^{[j]}\right) \varepsilon\right]<0
$$


That is,

$$
\eta^{[j]}(\varepsilon)=\sum_{i=1}^{\mathrm{N}}\left[\left(\pi_{i}^{[j]}\left|q_{i i}^{[j]}\right|+\varepsilon\right) \ln \left(\mu_{i}^{[j]}\right)+\left(\pi_{i}^{[j]}+\varepsilon\right)\left(-\lambda_{i}^{[j]}\right)\right]<0
$$

Based on (7), (8), and (A18), the following inequality should hold for $\forall t>0$

$$
\begin{aligned}
& \boldsymbol{x}^{T}(t) \boldsymbol{P}_{\sigma\left(t, \gamma_{k}\right)}^{\left[\gamma_{k}\right]} \boldsymbol{x}(t)<\boldsymbol{x}^{T}\left(t_{k}\right) \boldsymbol{P}_{\sigma\left(t_{k}, \gamma_{k}\right)}^{\left[\gamma_{k}\right]} \boldsymbol{x}\left(t_{k}\right) \times \exp \left\{\sum_{\sigma_{v}^{\gamma_{k}}=0}^{N_{\gamma_{k}}}\left(N_{\sigma_{v}^{\gamma_{k}}}^{\left[\gamma_{k}\right]}\left(t_{k}, t\right) \times \ln \mu_{\sigma_{v}^{\gamma_{k}}}^{\left[\gamma_{\gamma_{k}}\right]}\right)\right\} \times \\
& \exp \left\{-\int_{t_{k}}^{t} \lambda_{\sigma\left(s, \gamma_{k}\right)}^{\left[\gamma_{k}\right]} d s\right\}=x^{T}\left(t_{k}\right) \boldsymbol{P}_{\sigma\left(t_{k}, \gamma_{k}\right)}^{\left[\gamma_{k}\right]} \boldsymbol{x}\left(t_{k}\right) \times \exp \left\{\sum_{\sigma_{v}^{\gamma_{k}=0}}^{N}\left(N_{\sigma_{v}^{\gamma_{k}}}^{\left[\gamma_{k}\right]}\left(t_{k}, t\right) \times \ln \mu_{\sigma_{v}^{\gamma_{k}}}^{\left[\gamma_{k}\right]}\right)\right\} \times \\
& \exp \left\{\sum_{\sigma_{v}^{\gamma_{k}=0}}^{N_{\gamma_{k}}}\left(-\lambda_{\sigma_{v}^{\gamma_{k}}}^{\left[\gamma_{\gamma_{k}}\right]} \times T_{\sigma_{v}^{\gamma_{k}}}^{\left[\gamma_{k}\right]}\left(t_{k}, t\right)\right)\right\}<\boldsymbol{x}^{T}\left(t_{k}\right) \boldsymbol{P}_{\sigma\left(t_{k}, \gamma_{k}\right)}^{\left[\gamma_{k}\right]} \boldsymbol{x}\left(t_{k}\right) \times \\
& \exp \left\{\sum_{\sigma_{v}^{\gamma_{k}=0}}^{N}\left[\left(\left(\pi_{\sigma_{v}^{\gamma_{k}}}^{\left[\gamma_{k}\right]}\left|q_{\sigma_{v}^{\gamma_{k}} \sigma_{v}^{\gamma_{k}}}^{\left[\gamma_{k}\right]}\right|+\varepsilon\right) \times \ln \mu_{\sigma_{v}^{\gamma_{k}}}^{\left[\gamma_{k}\right]}\right)+\left(\pi_{\sigma_{v}^{\gamma_{k}}}^{\left[\gamma_{k}\right]}+\varepsilon\right) \times\left(-\lambda_{\sigma_{v}^{\gamma_{k}}}^{\left[\gamma_{k}\right]}\right)\right]\left(t-t_{k}\right)\right\} \\
& =\boldsymbol{x}^{T}\left(t_{k}\right) \boldsymbol{P}_{\sigma\left(t_{k}, \gamma_{k}\right)}^{\left[\gamma_{k}\right]} \boldsymbol{x}\left(t_{k}\right) \times \exp \left\{\eta^{\left[\gamma_{k}\right]}(\varepsilon) \times\left(t-t_{k}\right)\right\}
\end{aligned}
$$

Let $\left\{\left(0, \gamma_{0}\right),\left(t_{1}, \gamma_{1}\right), \ldots,\left(t_{k}, \gamma_{k}\right)\right\}$ denote the constructed SS of the switching signal determined deterministically by $\gamma(t)$ defined in $(H 4)$ and in $[0, T]$ The inequation in (A19) concludes that

$$
\begin{aligned}
& \mathrm{E}\left[\boldsymbol{x}^{T}(t) \boldsymbol{P}_{\sigma\left(t, \gamma_{k}\right)}^{\left[\gamma_{k}\right]} \boldsymbol{x}(t)\right]<\mathrm{E}\left(\boldsymbol{x}^{T}\left(t_{k}\right) \boldsymbol{P}_{\sigma\left(t_{k}, \gamma_{k}\right)}^{\left[\gamma_{k}\right]} \boldsymbol{x}\left(t_{k}\right)\right) \times \exp \left\{\eta^{\left[\gamma_{k}\right]}(\varepsilon) \times\left(t-t_{k}\right)\right\} \\
& <\mathrm{E}\left(\boldsymbol{x}^{T}\left(t_{k}\right) \boldsymbol{P}_{\sigma\left(t_{k}\right]}^{\left[\gamma_{k}\right)} \boldsymbol{x}\left(t_{k}\right)\right) \times \exp \left\{\eta^{\left[\gamma_{k}\right]}(\varepsilon) \times\left(t-t_{k}\right)\right\} \\
& <\mathrm{E}\left(\boldsymbol{x}^{T}\left(t_{k}\right) \boldsymbol{P}_{\sigma\left(t_{k}, \gamma_{k-1}\right)}^{\left[\gamma_{k-1}\right)} \boldsymbol{x}\left(t_{k}\right)\right) \times \exp \left\{\eta^{\left[\gamma_{k-1}\right]}(\varepsilon) \times\left(t_{k}-t_{k-1}\right)\right\} \times \exp \left\{\eta^{\left[\gamma_{k}\right]}(\varepsilon) \times\left(t-t_{k}\right)\right\} \\
& <\ldots \\
& <\mathrm{E}\left(\boldsymbol{x}_{0}^{T} \boldsymbol{P}_{\sigma\left(0, \gamma_{0}\right)}^{\left[\gamma_{0}\right]} \boldsymbol{x}_{0}\right) \times \exp \left\{\sum_{u=0}^{k} \eta^{\left[\gamma_{u}\right]}(\varepsilon) \times\left(t_{u+1}-t_{u}\right)\right\}
\end{aligned}
$$$$
\text { By (A18), supposing } \bar{\eta}(\varepsilon)=\max _{j \in \overline{\mathrm{M}}}\left\{\eta^{[j]}(\varepsilon)\right\}<0, \forall t>0 \text {. }
$$

$$
\mathrm{E}\left[\boldsymbol{x}^{T}(t) \boldsymbol{P}_{\sigma\left(t, \gamma_{k}\right)}^{\left[\gamma_{k}\right]} \boldsymbol{x}(t)\right]<\mathrm{E}\left(\boldsymbol{x}_{0}^{T} \boldsymbol{P}_{\sigma\left(0, \gamma_{0}\right)}^{\left[\gamma_{0}\right]} \boldsymbol{x}_{0}\right) \times \exp \{\bar{\eta}(\varepsilon) \times t\}
$$

It is evident that for any $x \in \boldsymbol{R}_{n}, i \in \overline{\mathrm{N}}, j \in \overline{\mathrm{M}}$, we have $\lambda_{1}\|\boldsymbol{x}\|^{2} \leq \boldsymbol{x}^{T} \boldsymbol{P}_{i}^{[j]} \boldsymbol{x} \leq \lambda_{2}\|\boldsymbol{x}\|^{2}$, where $\lambda_{1}=\min _{i \in \overline{\mathrm{N}}, j \in \overline{\mathrm{M}}}\left\{\lambda_{\min }\left(\boldsymbol{P}_{i}^{[j]}\right)\right\}>0$, and $\lambda_{2}=\max _{i \in \overline{\mathrm{N}}, j \in \overline{\mathrm{M}}}\left\{\lambda_{\max }\left(\boldsymbol{P}_{i}^{[j]}\right)\right\}>0$, $\lambda_{1}\|x\|^{2}, \lambda_{2}\|x\|^{2} \in K_{\infty}$.

Then

$$
\begin{aligned}
& \lambda_{1}\|x(t)\|^{2} \leq \mathrm{E}\left[\boldsymbol{x}^{T}(t) \boldsymbol{P}_{\sigma\left(t, \gamma_{k}\right)}^{\left[\gamma_{k}\right]} \boldsymbol{x}(t)\right]<\mathrm{E}\left[\boldsymbol{x}_{0}{ }^{T} \boldsymbol{P}_{\sigma\left(0, \gamma_{0}\right.}^{\left[\gamma_{0}\right]} \boldsymbol{x}_{0}\right] \times \exp \{\bar{\eta}(\varepsilon) \times t\} \leq \lambda_{2}\left\|x_{0}\right\|^{2} \times \exp \{\bar{\eta}(\varepsilon) \times t\} \\
& \|\boldsymbol{x}(t)\|^{2}<\frac{\lambda_{2}}{\lambda_{1}}\left\|\boldsymbol{x}_{0}\right\|^{2} \times \exp \{\bar{\eta}(\varepsilon) \times t\}, \mathrm{E}\left(\|\boldsymbol{x}(t)\|^{2}\right)<\frac{\lambda_{2}}{\lambda_{1}}\left\|\boldsymbol{x}_{0}\right\|^{2} \times \exp \{\bar{\eta}(\varepsilon) \times t\}
\end{aligned}
$$

The system in (1) is EMS stable by Definition 1.

\section{References}

1. Bolzern, P.; Colaneri, P.; Nicolao, G.D. Almost Sure Stability of Markov Jump Linear Systems with Deterministic Switching. IEEE Trans. Autom. Control 2013, 58, 209-214. [CrossRef]

2. Bolzern, P.; Colaneri, P.; Nicolao, G.D. Stochastic stability of Positive Markov Jump Linear Systems. Automatica 2014, 50, 1181-1187. [CrossRef]

3. Zhao, X.; Zhang, L.; Peng, S.; Liu, M. Stability of switched positive linear systems with average dwell time switching. Automatica 2012, 48, 1132-1137. [CrossRef]

4. Zhao, X.; Zhang, L.; Peng, S.; Liu, M. Stability and Stabilization of Switched Linear Systems with Mode-Dependent Average Dwell Time. IEEE Trans. Autom. Control 2012, 57, 1809-1815. [CrossRef]

5. Wang, G.; Liu, Y.; Lu, J.; Wang, Z. Stability analysis of totally positive switched linear systems with average dwell time switching. Nonlinear Anal. Hybrid Syst. 2020, 36, 100877. [CrossRef]

6. Ogura, M.; Preciado, V.M. Stability of Spreading Processes over Time-Varying Large-Scale Networks. IEEE Trans. Netw. Sci. Eng. 2015, 3, 44-57. [CrossRef]

7. Hespanha, J.P. Uniform stability of switched linear systems: Extensions of LaSalle's invariance principle. IEEE Trans. Autom. Control 2004, 49, 470-482. [CrossRef] 
8. Zhang, L.; Long, F. Stability Analysis for Dual Switching Linear Continuous-Time Systems. In Proceedings of the 38th China Control Conference, Guangzhou, China, 27-30 July 2019; Shanghai System Science Press: Shanghai, China, 2019; pp. 1154-1160.

9. Jie, Y.; Yang, S.; Yang, T.; Min, Z. Almost sure stability of a class of switched systems. In Proceedings of the 27th China Control \& Decision Conference, Qingdao, China, 23-26 May 2015; pp. 2093-2097.

10. Bolzern, P.; Colaneri, P.; De Nicolao, G. Design of stabilizing strategies for discrete-time dual switching linear systems. Automatica 2016, 69, 93-100. [CrossRef]

11. Pao, L.Y.; Johnson, K.E. Control of Wind Turbines. IEEE Control Syst. Mag. 2011, 31, 44-62.

12. Farina, L.; Rinaldi, S. Positive Linear Systems: Theory and Applications; John Wiley \& Sons: Hoboken, NJ, USA, 2000.

13. Ogura, M.; Preciado, V.M. Stability of Markov regenerative switched linear systems. Automatica 2016, 69, 169-175. [CrossRef]

14. Rami, M.A.; Tadeo, F.; Benzaouia, A. Control of constrained positive discrete systems. In Proceedings of the American Control Conference, New York, NY, USA, 9-13 July 2007.

15. Liu, X. Stability Analysis of Switched Positive Systems: A Switched Linear Copositive Lyapunov Function Method. IEEE Trans. Circuits Syst. II Express Briefs 2009, 56, 414-418.

16. May, R.M. Will a Large Complex System be Stable? Nature 1972, 238, 413. [CrossRef]

17. Jeffries, C.; Klee, V.; Pauline, V.D.D. When is a matrix sign stable? Can. J. Math. 1976, 29, 315-326. [CrossRef]

18. Yedavalli, R.K. Conditions for Hurwitz Stability/Instability of a Real Matrix via its Sign Pattern with a Necessary and Sufficient Condition for Magnitude Independent Stability. IFAC Papers OnLine 2018, 51, 663-667. [CrossRef]

19. Catral, M.; Olesky, D.D.; Driessche, P. Allow problems concerning spectral properties of sign pattern matrices: A survey. Linear Algebra Its Appl. 2009, 430, 3080-3094. [CrossRef]

20. Briat, C. Sign properties of Metzler matrices with applications. Linear Algebra Its Appl. 2016, 515, 53-86. [CrossRef]

21. Tong, W.; Chaoyang, D.; Qing, W.; Maolin, N. Sign Stability Analysis of Polytopic Uncertain Systems. Control. Theory Appl. 2015, $32,35-42$.

22. Qing, W.; Tong, W.; Yanze, H.; Dong, C. Sign Stability for Switched Linear Systems and Its Application in Flight Control. J. Appl. Math. 2014, 2014, 391617.

23. Yedavalli, R.K. Robust Control Design for Linear Systems Using an Ecological Sign-Stability Approach. J. Guid. Control Dyn. 2009, 32, 348-352. [CrossRef]

24. Yedavalli, R. Robust Satellite Attitude Control Using Qualitative Stability Concept from Ecology. In Proceedings of the AIAA Guidance, Navigation, \& Control Conference \& Exhibit, San Francisco, CA, USA, 15-18 August 2013.

25. Yedavalli, R.K.; Devarakonda, N. Sign-Stability Concept of Ecology for Control Design with Aerospace Applications. J. Guid. Control Dyn. 2015, 33, 333-346. [CrossRef]

26. Cavalcanti, J.; Balakrishnan, H. Sign-stability of Positive Markov Jump Linear Systems. Automatica 2019, 111, 108638. [CrossRef]

27. McCreesh, M.; Gharesifard, B. Stability of bounded subsets of Metzler sparse matrix cones. Automatica 2018, 95, 544-547. [CrossRef] 